\title{
A WKB Approximation of Elastic Waves Travelling on a Shell of Revolution
}

\author{
J. O. Morsbø1 ${ }^{1}$, S. V. Sorokin ${ }^{1}$, N. Peake ${ }^{2}$ \\ ${ }^{1}$ Aalborg University, Fibigerstrade 16, 9220 Aalborg East, Denmark \\ ${ }^{2}$ Cambridge University, DAMTP, Centre for Mathematical Sciences, Wilberforce Road, Cambridge, United Kingdom
}

\begin{abstract}
This paper is concerned with the elastic waveguide properties of an infinite pipe with circular cross section whose radius varies slowly along its length. The equations governing the elastodynamics of such shells are derived analytically, approximated asymptotically in the limit of slow axial variation, and solved by means of the WKB-method (WentzelKramers-Brillouin). From the derived solution the dispersion relation, modal coefficients, and wave amplification at each location along the structure are extracted, allowing identification of which types of waves are able to propagate along the structure at a given frequency. A key feature in the formulation of the model and the solution is that the radius and its variation are not specified in advance. Two characteristic examples of shells of revolution are presented to illustrate some general features of the waveguide properties, demonstrating how the evolution of the waves depends on the axial variation of the shell radius. It is explained how local resonances can be excited by the travelling waves and how strong amplifications of displacement can be produced. Specifically, for the axial/breathing wave it is shown that a local resonance is excited at the location where the frequency of the travelling wave and the radius of the shell exactly match the ring-frequency.
\end{abstract}

Keywords: WKB, dispersion diagram, waveguide, cylindrical shell, shell of revolution, elastodynamics, ring-frequency

\section{Introduction}

Structures containing shells of revolution include jet engines, wind turbine towers, piping systems, wind instruments, etc. Obviously structural vibration and noise emission, and therefore also the waveguide properties, are important topics in relation to many of these systems. The simplest example of a shell of revolution is a uniform cylinder. Wave propagation in and vibration of such shells is a classical subject covered in hundreds of research papers. Such a stateof-the-art is explained, on the one hand, by the obvious broadness of technical applications, where cylindrical shells are conveniently used and, on the other hand, by the relative simplicity of the mathematical modelling of the dynamics of shells of this kind. Specifically, the system of differential equations of the shell theory for a cylinder has constant coefficients, and, therefore, a general solution of these equations is of the simple exponential form. However, any departure from the geometry of a cylinder entails the spatial variability of Lamé parameters and curvatures preventing the determination of an exact solution. A solution of the problem of elastodynamics of an arbitrarily shaped shell, therefore, is usually obtained numerically and a variety of methods can be applied. There is one class of shells, however, for which an exact solution has the exponential form - but only in one of the coordinates, namely shells of revolution. It is conventional to derive equations of motion of such shells in the coordinate measured along the symmetry axis, that is the axial coordinate, and in the circumferential coordinate. Neither Lamé parameters nor radii of curvature of a shell of revolution are dependent upon the circumferential coordinate. Therefore, exponential functions of purely imaginary arguments in this coordinate are used to separate variables and to consider the $m$-spectra (with $m$ being the circumferential wavenumber) of vibration independent of each other. Nevertheless, the differential equations of motion of a shell of revolution have variable coefficients in the axial coordinate. To make progress with an analytical solution of these equations, some assumptions must be adopted regarding the shape of the shell. The most frequently used approximation is the initial imperfection of shape, which allows one to consider a shell of revolution as a perturbed cylindrical or conical shell, see [1], [2], and [3]. The common practice is to choose 
the shape of an imperfection and to introduce a small parameter of its amplitude (with reference to the radius of a perfect shell). To calculate eigenfrequencies, linearised equations of nonlinear vibrations of a cylindrical shell about deformed equilibrium configuration are used, [3]. Alternatively, Lamé parameters and radii of curvature are presented in the form of power series expansions on the imperfection parameter, and classical perturbation methods are used, [2]. All these references are concerned with eigenfrequencies of finite shells with various boundary conditions.

Quite surprisingly, very little attention seems to have been paid to the elastic wave propagation in shells of revolution. [4] studied waves travelling along a conical shell, and predicted how high-order modes successively cut off as they travel towards the narrow end of the shell. Unfortunately, the membrane shell theory adopted in this reference is insufficient to capture the global picture of the wave propagation in an elastic shell. Now, in this paper the restriction on the radius variation is different. Here the only initial assumption is that the radius variation happens slowly. This means that the slope of the radius as a function of the axial coordinate is small. The slowly varying radius permits the application of the so-called WKB method (Wentzel-Kramers-Brillouin). The WKB method has previously been used to model the properties of curved waveguides, see [5] and [6]. Moreover, it the pasthis been used by [7] (though without designating it as the WKB method) to analyse vibrations in a finite length shell of revolution. However, by using the WKB method to study the waveguide properties of an infinite shell of revolution it is possible to gain insight into how the dispersion relation, the modal vector, and the amplitude are affected by a given radius variation. It is exactly this insight which is regarded as the novelty of this work. In this connection it should though be emphasised that turning point analysis is outside the scope of this paper. Moreover, it is also fair to mention that the WKB method in principle is similar to the two-time scale method which is used for problems depending on multiple time scales. The only major difference is the change of coordinates.

\section{Problem Formulation and Validation}

In what follows a model to determine the waveguide properties of a shell of revolution will be developed and solved. The solution will be validated by calculating the corresponding energy flux from Hamilton's principle and checking that it is conserved along the waveguide. In this way it is also possible to keep track of the kinetic and potential energy distribution along the waveguide.

\subsection{Modelling Scheme}

The model takes its origin in classical thin shell theory. From this a set of governing partial differential equations (PDEs) is obtained and solved asymptotically by means of the WKB method. The applied shell theory was originally presented by [8], which is a linear theory founded on classical Kirchhoff-Love kinematics. Apart from omitting a few terms of higher order of smallness, it is identical to the perhaps more well-known theory by [9], though, as pointed out by [8], the contribution of these small terms is beyond the uncertainty caused by the basic Kirchhoff-Love assumptions, and thus it is more consistent to omit them.

\subsubsection{Shell of Revolution}
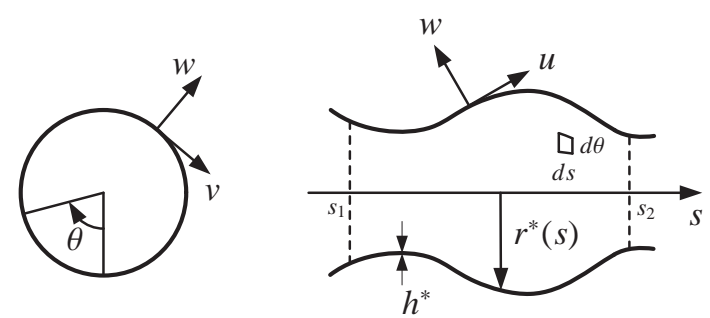

Figure 1: The geometry of the shell's mid-surface is composed of the shell thickness, $h^{*}$, and the radius, $r^{*}(s)$, while each point on the shell can be uniquely identified by $(s, \theta)$. The displacements $u, v$, and $w$ are: Axial displacement, circumferential displacement, and normal displacement.

The general shell theory of [8] establishes three groups of relations: The displacement-deformation relations, the constitutive relations, and the equilibrium equations, which within the framework of structural dynamics are known 
as the equations of motion. The equations of motion are expressed in terms of force and moment resultants, but by combining with the constitutive relations and the displacement-deformation relations, the equations of motion are expressed in terms of displacements instead. Although the equations become more complicated in this way, it makes it possible to formulate a solution which is intuitively easier to relate to, as it gives expressions for the actual displacements. However, to specialise the general equations of motion to the shell of revolution, like the one illustrated in figure 1 , the dependency on the circumferential coordinate, $\theta$, is removed from the Lamé parameters and from the radii of curvature. When this has been done the equations presented in appendix A are obtained.

The equations in appendix A are expressed in terms of measurable dimensional quantities such as the shell thickness, radius, Young's modulus, etc. But to generalise the results as well as making the equations asymptotically consistent it is useful to non-dimensionalise the system by proper scaling. Thus, it is assumed that the shape of the mid-surface can be formulated in the two non-dimensional variables, $s$ and $\theta$, in a parametrisation of the form $\mathbf{r}^{*}(s, \theta)=r_{0}^{*}\left[f_{1}(s, \theta), f_{2}(s, \theta), f_{3}(s, \theta)\right]^{T}$, where quantities marked with "*” are dimensional, and $r_{0}^{*}$ is some characteristic radius somewhere along the shell. In this case the non-dimensional Lamé parameters and radii of curvature will be scaled as $A_{i}^{*}=r_{0}^{*} A_{i}$ and $R_{i}^{*}=r_{0}^{*} R_{i}$. Likewise the shell thickness is scaled by introducing the thickness parameter $\delta=\frac{h^{*}}{r_{0}^{*}}$ and the frequency and displacements are scaled by assuming that the solution take the form

$$
\mathbf{u}^{*}\left(s, \theta, t^{*}\right)=\left[\begin{array}{c}
u^{*}\left(s, \theta, t^{*}\right) \\
v^{*}\left(s, \theta, t^{*}\right) \\
w^{*}\left(s, \theta, t^{*}\right)
\end{array}\right]=r_{0}^{*}\left[\begin{array}{c}
\tilde{u}(s, \theta) \\
\tilde{v}(s, \theta) \\
\tilde{w}(s, \theta)
\end{array}\right] e^{\frac{i \omega c^{*} t^{*}}{r_{0}^{*}}}=r_{0}^{*} \tilde{\mathbf{u}}(s, \theta) e^{\frac{i \omega c^{*} t^{*}}{r_{0}^{*}}},
$$

where $\omega$ is the frequency and $c^{*} \equiv \sqrt{E^{*} / \rho^{*}}$. In this way the system of PDEs governing the dynamics of the shell of revolution can be written as:

$$
\left(\mathbf{L}+\left(1-v^{2}\right) \omega^{2}\right) \tilde{\mathbf{u}}=\mathbf{0},
$$

where $\mathbf{L}$ is a $3 \times 3$ partial differential operator and $v$ is Poisson's ratio.

A general solution to this equation, for any imaginable shell of revolution, hardly exists. Instead, to ease the problem, the focus in the following will be on a sub-set of these shells which is characterised by a slowly varying radius compared to the length of the waves travelling along the shell. In other words, by assuming that within the order of one wavelength the change of radius can be neglected, so that the wave will locally see the shell of revolution as a uniform cylinder with the local properties. However, as the wave propagates over longer distances the effects of the changing radius will be accumulated in the wave, affecting wavelength, mode shape, and amplitude. As will be demonstrated, for such a sub-set of shells the WKB method turns out to be very useful.

\subsubsection{WKB Solution}

The WKB method is an asymptotic method which simplifies a more general problem by exploiting the smallness of some characterising parameter. In this context, where the radius is assumed to vary slowly, a small parameter, $\epsilon$, could be defined as the ratio between $r_{0}^{*}$ and some distance, $D^{*}$, over which the radius varies perceptibly. With this parameter the axial coordinate, $s$, can be regarded as a so-called fast coordinate while a slow coordinate, $S$, is defined as:

$$
\frac{r_{0}^{*}}{D^{*}} s=\epsilon S=S \quad \text { where: } \epsilon \ll 1
$$

In this connection the small parameter, $\epsilon$, will be designated as the slow-scale parameter. At this stage, nothing has been assumed about how the radius varies, except that it changes slowly on the scale of the wavelength. The mid-surface can be parameterised as:

$$
\mathbf{r}^{*}(s, \theta)=r_{0}^{*}\left[\begin{array}{c}
s \\
r(S) \cos (\theta) \\
r(S) \sin (\theta)
\end{array}\right]
$$


where $r(S)$ is the radius function. It should be remarked that the first component of the parametrisation is just the fast coordinate multiplied by $r_{0}^{*}$, while the radius function depends on the slow coordinate. Furthermore, in order to have physical meaning, the radius function must be real valued, continuous, and, and in accordance with [8], not give rise to a principal radius of curvature violating $\max \left\{\frac{\delta}{R_{i}}\right\} \leq \frac{1}{20}$ in order to follow the fundamental assumptions of the thin shell theory. With this parametrisation it may also be seen that $r_{0}^{*}$ is simply the measurable radius exactly where $r(S)=1$. With this parametrisation, and by means of basic differential geometry, the Lamé parameters and the two principal radii of curvature are determined as:

$$
\begin{array}{ll}
A_{s}=\sqrt{1+\epsilon^{2} r^{\prime}(S)^{2}} & A_{\theta}=r(S) \\
R_{S}=\frac{\left(1+\epsilon^{2} r^{\prime}(S)^{2}\right)^{\frac{3}{2}}}{\epsilon^{2} r^{\prime \prime}(S)} & R_{\theta}=-r(S) \sqrt{1+\epsilon^{2} r^{\prime}(S)^{2}} .
\end{array}
$$

These parameters may be substituted into the governing equations of the general shell of revolution, while remembering that due to equation (3) $\frac{\partial}{\partial S}=\epsilon \frac{\partial}{\partial S}$.

Taking the complexity of the equations presented in appendix A and the functions in (5) into account, the differential operator, $\mathbf{L}$, become rather cumbersome. But fortunately it is possible to simplify $\mathbf{L}$ considerably by asymptotic approximation. In its non-dimensional form $\mathbf{L}$ only depends on the slow-scale parameter, the thickness parameter, Poisson's ratio, and the radius function along with some of its derivatives. Of course, the thickness parameter is small, and in principle this make both the slow-scale parameter and the thickness parameter candidates for asymptotic expansion. One of these small parameters could be eliminated by introducing some relation between them, and then expanding the equations in a single small parameter. On the other hand, the aim here is to keep these parameters completely independent and to use the slowness of the radius variation as a means to control the accuracy of the approximation. Thus equation (2) can be expanded as:

$$
\left(\mathbf{L}_{0}+\epsilon \mathbf{L}_{1}+O\left(\epsilon^{2}\right)+\left(1-v^{2}\right) \omega^{2}\right) \tilde{\mathbf{u}}=\mathbf{0} .
$$

Within the framework of the standard WKB method it is enough only to consider $O(1)$ - and $O(\epsilon)$-terms. The components of $\mathbf{L}_{0}$ are found to be:

$$
\begin{aligned}
L_{0,11} & =\frac{\partial^{2}}{\partial s^{2}}+\frac{1-v}{2 r(S)^{2}} \frac{\partial^{2}}{\partial \theta^{2}} \\
L_{0,12} & =\frac{1+v}{2 r(S)} \frac{\partial^{2}}{\partial s \partial \theta} \\
L_{0,13} & =-\frac{v}{r(S)} \frac{\partial}{\partial s}
\end{aligned}
$$

$$
\begin{aligned}
& L_{0,21}=L_{0,12} \\
& L_{0,22}=\left(\frac{1-v}{2}+\frac{(1-v) \delta^{2}}{6 r(S)^{2}}\right) \frac{\partial^{2}}{\partial s^{2}}+\left(\frac{1}{r(S)^{2}}+\frac{\delta^{2}}{12 r(S)^{4}}\right) \frac{\partial^{2}}{\partial \theta^{2}} \\
& L_{0,23}=\frac{\delta^{2}}{12 r(S)^{4}} \frac{\partial^{3}}{\partial \theta^{3}}+\frac{(2-v) \delta^{2}}{12 r(S)^{2}} \frac{\partial^{3}}{\partial s^{2} \partial \theta}-\frac{1}{r(S)^{2}} \frac{\partial}{\partial \theta} \\
& L_{0,31}=-L_{0,13} \\
& L_{0,32}=L_{0,23} \\
& L_{0,33}=\frac{\delta^{2}}{12} \frac{\partial^{4}}{\partial s^{4}}+\frac{\delta^{2}}{12 r(S)^{4}} \frac{\partial^{4}}{\partial \theta^{4}}+\frac{\delta^{2}}{6 r(S)^{2}} \frac{\partial^{4}}{\partial s^{2} \theta^{2}}+\frac{1}{r(S)^{2}},
\end{aligned}
$$

and the components of $\mathbf{L}_{1}$ are: 


$$
\begin{aligned}
& L_{1,11}=\frac{r^{\prime}(S)}{r(S)} \frac{\partial}{\partial s} \\
& L_{1,12}=-\frac{(3-v) r^{\prime}(S)}{2 r(S)^{2}} \frac{\partial}{\partial \theta} \\
& L_{1,13}=\frac{r^{\prime}(S)}{r(S)^{2}} \\
& L_{1,21}=-L_{1,12} \\
& L_{1,22}=\left(\frac{(1-v) r^{\prime}(S)}{2 r(S)}-\frac{(1-v) \delta^{2} r^{\prime}(S)}{6 r(S)^{3}}\right) \frac{\partial}{\partial s} \\
& L_{1,23}=-\frac{(1-2 v) \delta^{2} r^{\prime}(S)}{12 r(S)^{3}} \frac{\partial^{2}}{\partial s \partial \theta} \\
& L_{1,31}=L_{1,13} \\
& L_{1,32}=-\frac{\delta^{2} r^{\prime}(S)}{4 r(S)^{3}} \frac{\partial^{2}}{\partial s \partial \theta} \\
& L_{1,33}=\frac{\delta^{2} r^{\prime}(S)}{6 r(S)} \frac{\partial^{3}}{\partial s^{3}}-\frac{\delta^{2} r^{\prime}(S)}{6 r(S)^{3}} \frac{\partial^{3}}{\partial s \partial \theta^{2}} .
\end{aligned}
$$

A closer inspection of these operators reveals that the leading order operator, $\mathbf{L}_{0}$, is identical to the case of the uniform cylinder, but properly corrected by $r(S)$. The next step is to pose the standard WKB expansion:

$$
\begin{aligned}
\tilde{\mathbf{u}}(S, \theta) & =\sum_{m=0}^{\infty} e^{i m \theta}\left(\boldsymbol{\alpha}_{0}(S)+\epsilon \boldsymbol{\alpha}_{1}(S)+O\left(\epsilon^{2}\right)\right) e^{\frac{i}{\epsilon} \int_{0}^{S} k(\sigma) d \sigma} \\
& =\sum_{m=0}^{\infty} e^{i m \theta}\left(\left[\begin{array}{l}
\alpha_{0}(S) \\
\beta_{0}(S) \\
\gamma_{0}(S)
\end{array}\right]+\epsilon\left[\begin{array}{l}
\alpha_{1}(S) \\
\beta_{1}(S) \\
\gamma_{1}(S)
\end{array}\right]+O\left(\epsilon^{2}\right)\right) e^{\frac{i}{\epsilon} \int_{0}^{S} k(\sigma) d \sigma},
\end{aligned}
$$

where $m$ is the circumferential wavenumber, $\boldsymbol{\alpha}_{i}$ is the modal vector related to $O\left(\epsilon^{i}\right)$, and $k$ is the axial wavenumber. This solution is separable in $S$ and $\theta$ due to the symmetry of the shell of revolution. In the circumferential direction the solution is no different from the corresponding solution of a uniform cylindrical shell. Because the shell is closed in that direction, it only supports waves having a period of $2 \pi$. Any such wave can be decomposed into a Fourier series. In the meantime the coefficients of the governing differential equations are independent of $\theta$. Again, as in cylindrical shell theory, this has the consequence that the waves associated with different circumferential wavenumber are completely uncoupled from each other. Therefore the solution can be studied for just a single circumferential wavenumber at a time, instead of the whole infinite sum at once. In the axial direction the effects of the slowly varying radius are taken into account. Thus the modal vector may be a function of $S$ and, in order to be asymptotically consistent, it is expanded in powers of $\epsilon$ as in equation (6). Also the axial wavenumber is affected by the changing radius, and following [10] this is handled by an integral formulation as the changing axial wavenumber is accumulated in the wave as it propagates along the shell.

By substitution of equation (7) into equation (6) and collecting $O(1)$-terms and $O(\epsilon)$-terms, the following two system of equations are found:

$$
\begin{aligned}
& O(1): \quad \mathbf{M} \boldsymbol{\alpha}_{0}=\mathbf{0} \\
& O(\epsilon): \quad \mathbf{M} \boldsymbol{\alpha}_{1}=\mathbf{f},
\end{aligned}
$$


where the components of $\mathbf{M}$ are:

$$
\begin{aligned}
& M_{11}=-k(S)^{2}-\frac{(1-v) m^{2}}{2 r(S)^{2}}+\left(1-v^{2}\right) \omega^{2} \\
& M_{12}=-\frac{(1+v) m k(S)}{2 r(S)} \\
& M_{13}=-\frac{i v k(S)}{r(S)} \\
& M_{21}=M_{12} \\
& M_{22}=-\frac{(1-v) k(S)^{2}}{2}-\frac{(1-v) \delta^{2} k(S)^{2}}{6 r(S)^{2}}-\frac{m^{2}}{r(S)^{2}}-\frac{\delta^{2} m^{2}}{12 r(S)^{4}}+\left(1-v^{2}\right) \omega^{2} \\
& M_{23}=-\frac{i \delta^{2} m^{3}}{12 r(S)^{4}}-\frac{i(2-v) \delta^{2} m k(S)^{2}}{12 r(S)^{2}}+\frac{i m}{r(S)^{2}} \\
& M_{31}=M_{13} \\
& M_{32}=M_{23} \\
& M_{33}=\frac{\delta^{2} k(S)^{4}}{12}+\frac{\delta^{2} m^{4}}{12 r(S)^{4}}+\frac{\delta^{2} m^{2} k(S)^{2}}{6 r(S)^{2}}+\frac{1}{r(S)^{2}}-\left(1-v^{2}\right) \omega^{2},
\end{aligned}
$$

and the components of $\mathbf{f}$ are:

$$
\begin{aligned}
f_{1}= & i k^{\prime}(S) \alpha_{0}(S)+\frac{i k(S) r^{\prime}(S) \alpha_{0}(S)}{r(S)}-\frac{i(3-v) m r^{\prime}(S) \beta_{0}(S)}{2 r(S)^{2}}+\frac{r^{\prime}(S) \gamma_{0}(S)}{r(S)^{2}}+2 i k(S) \alpha_{0}^{\prime}(S)+\frac{i(1+v) m \beta_{0}^{\prime}(S)}{2 r(S)}-\frac{v \gamma_{0}^{\prime}(S)}{r(S)} \\
f_{2}= & \frac{i(3-v) m r^{\prime}(S) \alpha_{0}(S)}{2 r(S)^{2}}+\frac{i(1-v)}{2}\left(\frac{r^{\prime}(S) k(S)}{r(S)}-\frac{\delta^{2} r^{\prime}(S) k(S)}{3 r(S)^{3}}+k^{\prime}(S)+\frac{\delta^{2} k^{\prime}(S)}{3 r(S)^{2}}\right) \beta_{0}(S)+ \\
& +\frac{m \delta^{2}}{12 r(S)^{2}}\left(\frac{(1-2 v) r^{\prime}(S) k(S)}{r(S)}-(2-v) k^{\prime}(S)\right) \gamma_{0}(S)+ \\
& +\frac{i(1+v) m \alpha_{0}^{\prime}(S)}{2 r(S)}+i(1-v)\left(k(S)+\frac{\delta^{2} k(S)}{3 r(S)^{2}}\right) \beta_{0}^{\prime}(S)-\frac{(2-v) m \delta^{2} k(S)}{6 r(S)^{2}} \gamma_{0}^{\prime}(S) \\
f_{3}= & -\frac{r^{\prime}(S) \alpha_{0}(S)}{r(S)^{2}}-\frac{m \delta^{2}}{12 r(S)^{2}}\left((2-v) k^{\prime}(S)-\frac{3 r^{\prime}(S) k(S)}{r(S)}\right) \beta_{0}(S)-\frac{i \delta^{2}}{2}\left(\frac{r^{\prime}(S) k(S)^{3}}{3 r(S)}-\frac{m^{2} r^{\prime}(S) k(S)}{3 r(S)^{3}}+\right. \\
& \left.+\frac{m^{2} \delta^{2} k^{\prime}(S)}{3 r(S)^{2}}+k^{\prime}(S) k(S)^{2}\right) \gamma_{0}(S)-\frac{v \alpha_{0}^{\prime}(S)}{r(S)}-\frac{(2-v) m \delta^{2} k(S) \beta_{0}^{\prime}(S)}{6 r(S)^{2}}-\frac{i \delta^{2}}{3}\left(k(S)^{3}+\frac{m^{2} k(S)}{r(S)^{2}}\right) \gamma_{0}^{\prime}(S) .
\end{aligned}
$$

Starting out with the $O(1)$-system, the leading order dispersion relation can be extracted by taking the determinant and equating it to zero, i.e. $|\mathbf{M}|=0$, while the modal vector, $\boldsymbol{\alpha}_{0}$, except for a special case where $m=0$ (this particular case will be treated in the following subsection), can be written as:

$$
\boldsymbol{\alpha}_{0}=\left[\begin{array}{c}
\alpha_{0}(S) \\
\beta_{0}(S) \\
\gamma_{0}(S)
\end{array}\right]=\left[\begin{array}{c}
1 \\
V(S) \\
W(S)
\end{array}\right] \alpha_{0}(S),
$$


where the modal coefficients $V(S)$ and $W(S)$ can be determined from the first and third rows in equation (8a) as

$$
\begin{aligned}
& V(S)=\frac{M_{13}^{2}-M_{11} M_{33}}{M_{12} M_{33}-M_{13} M_{23}} \\
& W(S)=\frac{M_{11} M_{23}-M_{12} M_{13}}{M_{12} M_{33}-M_{13} M_{23}} .
\end{aligned}
$$

In this way $\alpha_{0}(S)$ will act as the amplitude of the modal vector. Provided that the dispersion relation is obeyed, any arbitrary choice of $\alpha_{0}(S)$ will, together with the stated modal coefficients, solve (8a). Such a leading order solution contains information about how the axial wavenumber depends on the radius function and how the modal vector changes direction. However, to determine how the amplification of the modal vector also depends on the radius function, and thereby determine $\alpha_{0}(S)$, the $O(\epsilon)$-system must be considered. From the two equation systems in (8) it can be noticed that $\mathbf{M}$ appears in both systems. It can also be noticed that the components of the $O(1)$ modal vector, i.e. $\alpha_{0}(S), \beta_{0}(S), \gamma_{0}(S)$, and their derivatives appear in $\mathbf{f}$. This indicates that some relation between the two systems might exist. Hence, by realising that $\mathbf{M}$ is a self-adjoint matrix ${ }^{1}$ one way forward is to consider the inner product, written as $\langle\cdot, \cdot\rangle$, of $\boldsymbol{\alpha}_{0}$ and $\mathbf{f}$ :

$$
\begin{aligned}
\left\langle\boldsymbol{\alpha}_{0}, \mathbf{f}\right\rangle & =\left\langle\boldsymbol{\alpha}_{0}, \mathbf{M} \boldsymbol{\alpha}_{1}\right\rangle \\
& =\left\langle\mathbf{M}^{\dagger} \boldsymbol{\alpha}_{0}, \boldsymbol{\alpha}_{1}\right\rangle \\
& =\left\langle\mathbf{0}, \boldsymbol{\alpha}_{1}\right\rangle \equiv 0,
\end{aligned}
$$

and because $\alpha_{0}$ and $\mathbf{f}$ are complex vectors the inner product is defined as

$$
\begin{aligned}
0=\left\langle\boldsymbol{\alpha}_{0}, \mathbf{f}\right\rangle & \equiv \boldsymbol{\alpha}_{0} \cdot \overline{\mathbf{f}} \\
& =\alpha_{0}(S) \overline{f_{1}}+\beta_{0}(S) \overline{f_{2}}+\gamma_{0}(S) \overline{f_{3}} .
\end{aligned}
$$

This scalar equation is known as the solvability condition for the $O(\epsilon)$-system. From the general modal vector in equation (9) it is seen that $\beta_{0}(S)=V(S) \alpha_{0}(S) \Rightarrow \beta_{0}^{\prime}(S)=V^{\prime}(S) \alpha_{0}(S)+V(S) \alpha_{0}^{\prime}(S)$ and $\gamma_{0}(S)=W(S) \alpha_{0}(S) \Rightarrow$ $\gamma_{0}^{\prime}(S)=W^{\prime}(S) \alpha_{0}(S)+W(S) \alpha_{0}^{\prime}(S)$. With these identities $\beta_{0}(S), \beta_{0}^{\prime}(S), \gamma_{0}(S)$, and $\gamma_{0}^{\prime}(S)$ can be eliminated from the solvability condition, as it is presented above (remembering also to eliminate these quantities from $\overline{f_{1}}, \overline{f_{2}}$, and $\overline{f_{3}}$ ). After that, by collecting the coefficients in front of, respectively, $\alpha_{0}(S)$ and $\alpha_{0}^{\prime}(S)$ the solvability condition can then be rewritten as

$$
P(S) \alpha_{0}^{\prime}(S)+Q(S) \alpha_{0}(S)=0
$$

where $P(S)$ and $Q(S)$ are ordinary scalar functions. This equation has the general solution

$$
\alpha_{0}(S)=C e^{-\int \frac{Q(S)}{P(S)} d S}
$$

where $C$ is some arbitrary constant. Thus, finding $\alpha_{0}(S)$ becomes a matter of extracting $P(S)$ and $Q(S)$ from the solvability condition and integrating their ratio.

\footnotetext{
${ }^{1}$ A self-adjoint, or Hermitian, matrix is equal to the conjugate transposed of its own: $\mathbf{A}=\mathbf{A}^{\dagger}$
} 


\subsubsection{When $m=0$ - The Axial, Torsional, and Breathing Wave Mode}

If the circumferential wavenumber $m=0$, the equations become considerably simpler, and in this case the modal vector in equation (9) and its coefficients are not needed. This circumferential wavenumber is associated with wave modes which have a uniform displacement along the circumference in direction of the $u-, v-$, or $w$-axis, cf. figure 1. Such wave modes are recognised as the axial mode, the torsional mode, and the breathing mode. In this case $\mathbf{M}$ simplifies to:

$$
\mathbf{M}=\left[\begin{array}{ccc}
-k(S)^{2}+\left(1-v^{2}\right) \omega^{2} & 0 & -\frac{i v k(S)}{r(S)} \\
0 & -\frac{(1-v) k(S)^{2}}{2}-\frac{(1-v) \delta^{\delta^{2} k(S)^{2}}}{6 r(S)^{2}}+\left(1-v^{2}\right) \omega^{2} & 0 \\
M_{13} & 0 & \frac{\delta^{2} k(S)^{4}}{12}+\frac{1}{r(S)^{2}}-\left(1-v^{2}\right) \omega^{2}
\end{array}\right] .
$$

The fact that $M_{22}$ uncouples from the other components indicates that the corresponding $v$-displacement can be studied independently from the other displacements. Thus the wavenumber of the associated torsional wave mode can be determined by simply setting $M_{22}=0$, giving

$$
k_{t}(S)=\frac{\sqrt{6(1+v)} \omega r(S)}{\sqrt{\delta^{2}+3 r(S)^{2}}} \simeq \sqrt{2(1+v)} \omega,
$$

where the last simplification relies on the fundamental limitation of the applied thin shell theory, namely that $20 \delta \leq$ $r(S)$. Furthermore, the equivalent modal vector will be $\alpha_{0}=\left[0, \beta_{t}(S), 0\right]^{T}$, and thus the solvability condition in (12) reduces to the product of $\beta_{t}(S)$ and $\overline{f_{2}}$. This product take the same form as (13), but with $\beta_{t}(S)$ as unknown. The ratio between $Q_{t}(S)$ and $P_{t}(S)$ then becomes:

$$
\frac{Q_{t}(S)}{P_{t}(S)}=\frac{\delta^{2}\left(k_{t}^{\prime}(S) r(S)-k_{t}(S) r^{\prime}(S)\right)+3\left(k_{t}^{\prime}(S) r(S)^{3}+k_{t}(S) r(S)^{2} r^{\prime}(S)\right)}{2 \delta^{2} k_{t}(S) r(S)+6 k_{t}(S), r(S)^{3}},
$$

which integrates to

$$
\int \frac{Q_{t}(S)}{P_{t}(S)} d S=\ln \left(\frac{\sqrt{k_{t}(S)} \sqrt{\delta^{2}+3 r(S)^{2}}}{\sqrt{r(S)}}\right)
$$

In this way the amplitude of the torsion mode becomes

$$
\beta_{t}(S)=C_{t} \frac{\sqrt{r(S)}}{\sqrt{k_{t}(S)} \sqrt{\delta^{2}+3 r(S)^{2}}} \simeq C_{t} \frac{1}{\sqrt{3 k_{t}(S) r(S)}} .
$$

Not only does the expression for the torsional wavenumber and amplitude turn out to be rather simple, but they also show that as long as the thickness parameter is small and the radius function varies slowly, then the wavenumber just depends on Poisson's ratio and the frequency, while the amplitude depends on the axial coordinate through the factor $\frac{1}{\sqrt{r(S)}}$.

In a similar way, the modal vector and the amplitude for the axial mode and the breathing mode are determined. But due to Poisson coupling the axial displacement and normal displacement are linked via $M_{13}$, meaning that, except for the special case where $v=0$, these waves will inevitably excite each other. Consequently it may be a question of definitions whether the combined mode is designated as the axial mode or the breathing mode, or just as the axial/breathing mode. Their common wavenumber is determined by solving the dispersion relation, $M_{11} M_{33}-M_{13}^{2}=$ 0 , which will be a sixth order polynomial. Because the powers of the wavenumber are all even the roots of the polynomial can be determined analytically as an implicit third order polynomial. In the meantime these analytical roots become very cumbersome, and therefore it may be more practical to solve it numerically. The modal vector, on the other hand, can simply be written as 


$$
\boldsymbol{\alpha}_{0}=\left[\begin{array}{c}
1 \\
0 \\
-\frac{M_{11}}{M_{13}}
\end{array}\right] \alpha_{a}(S)=\left[\begin{array}{c}
1 \\
0 \\
-\frac{i r(S)\left(k_{a}(S)^{2}-\left(1-\nu^{2}\right) \omega^{2}\right)}{v k_{a}(S)}
\end{array}\right] \alpha_{a}(S) .
$$

$Q_{a}(S)$ and $P_{a}(S)$ of equation (13) then become:

$$
\begin{aligned}
Q_{a}(S)= & \frac{i \delta^{2}\left(1-v^{2}\right)^{2} \omega^{4}}{v^{2}}\left(\frac{1}{6} k_{a}^{\prime}(S) r(S)^{2}+\frac{1}{2} k_{a}(S) r(S) r^{\prime}(S)\right)- \\
& -i\left(1-v^{2}\right) \omega^{2}\left(\frac{\delta^{2}}{v^{2}}\left(k_{a}(S)^{2} k_{a}^{\prime}(S) r(S)^{2}+k_{a}(S)^{3} r(S) r^{\prime}(S)\right)+\frac{k_{a}^{\prime}(S)}{k_{a}(S)^{2}}-\frac{r^{\prime}(S)}{k_{a}(S) r(S)}\right)+ \\
& +\frac{i \delta^{2}}{v^{2}}\left(\frac{5}{6} k_{a}(S)^{4} k_{a}^{\prime}(S) r(S)^{2}+\frac{1}{2} k_{a}(S)^{5} r(S) r^{\prime}(S)\right) \\
P_{a}(S)= & \frac{i \delta^{2}\left(1-v^{2}\right)^{2} \omega^{4}}{3 v^{2}} k_{a}(S) r(S)^{2}-i\left(1-v^{2}\right) \omega^{2}\left(\frac{2 \delta^{2}}{3 v^{2}} k_{a}(S)^{3} r(S)^{2}-2 \frac{1}{k_{a}(S)}\right)+\frac{i \delta^{2}}{3 v^{2}} k_{a}(S)^{5} r(S)^{2} .
\end{aligned}
$$

Despite the complexity of $Q_{a}(S)$ and $P_{a}(S)$ their ratio may be integrated analytically. Thus, using equation (14), the modal vector consists of the following components:

$$
\begin{aligned}
& \alpha_{a}(S)=C_{a} \frac{\sqrt{k_{a}(S)}}{\sqrt{r(S)} \sqrt{6 v^{2}\left(1-v^{2}\right) \omega^{2}+\left(\delta\left(\left(1-v^{2}\right) \omega^{2}+k_{a}(S)^{2}\right) k_{a}(S) r(S)\right)^{2}}} \\
& \gamma_{a}(S)=C_{a} \frac{i \sqrt{r(S)}\left(k_{a}(S)^{2}-\left(1-v^{2}\right) \omega^{2}\right)}{v \sqrt{k_{a}(S)} \sqrt{6 v^{2}\left(1-v^{2}\right) \omega^{2}+\left(\delta\left(\left(1-v^{2}\right) \omega^{2}+k_{a}(S)^{2}\right) k_{a}(S) r(S)\right)^{2}}} .
\end{aligned}
$$

These expressions cannot be simplified further as easily as in the torsion case. Even though $\delta^{2}$ is a small number it is in the denominator multiplied with an $O\left(k_{a}(S)^{6}\right)$-function. And as it will be demonstrated later, $k_{a}(S)$ can indeed take values of more than 10 , meaning that $\delta^{2} \cdot k_{a}(S)^{6}$ can be a significant number.

\subsubsection{When $m=0-$ In Relation to Conventional Beam and Rod Theory}

The wave modes associated with $m=0$ have some noticeable similarities with what can be found via conventional beam and rod theory. The simplest example is the torsional mode in a rod with slowly varying radius. The partial differential equation governing this mode is, see [11],

$$
0=\frac{\partial}{\partial s^{*}}\left(G^{*} J^{*}(S) \frac{\partial \theta}{\partial s^{*}}\right)-I_{o}^{*}(S) \frac{\partial^{2} \theta}{\partial t^{* 2}},
$$

where $G^{*}$ is the shear modulus, $J^{*}(S)$ is the polar moment of inertia, and $I_{o}^{*}(S)$ is the polar mass moment of inertia per unit length. By letting $G^{*}=\frac{E^{*}}{2(1+v)}, J^{*}(S)=2 \pi r_{0}^{*} h^{*}\left(r_{0}^{*} r(S)\right)^{3}, I_{o}^{*}(S)=2 \pi \rho^{*} r_{0}^{*} h^{*}\left(r_{0}^{*} r(S)\right)^{3}, \theta=\frac{v}{r(S)}$, and using the same time dependency as for the shell solution, i.e. $v^{*}\left(s, t^{*}\right)=r_{0}^{*} \tilde{v}(s) \operatorname{Exp}\left(\frac{i \omega c^{*} t^{*}}{r_{0}^{*}}\right)$, while recalling that $\frac{\partial}{\partial s^{*}}=\frac{1}{r_{0}^{*}} \frac{\partial}{\partial s}$, then the corresponding non-dimensional differential equation becomes

$$
0=\frac{\partial}{\partial s}\left(r(S)^{3} \frac{\partial}{\partial s}\left(\frac{\tilde{v}}{r(S)}\right)\right)+2(1+v) \omega^{2} r(S)^{2} \tilde{v}
$$


By substituting the WKB solution $\tilde{v}(s)=\left(\beta_{0}(S)+\epsilon \beta_{1}(S)\right) \operatorname{Exp}\left(\frac{i}{\epsilon} \int_{0}^{S} k(\sigma) d \sigma\right)$ and collecting $O(1)$-terms and $O(\epsilon)$ terms, the wavenumber can be determined from the $O(1)$-system and the amplitude can be determined from the $O(\epsilon)$-system. Thus, the wavenumber of the torsional wave in the $\operatorname{rod}$ is $k_{t}(S)=\sqrt{2(1+v)} \omega$ while the amplitude is $\beta_{t}(S)=(\sqrt{k(S)} \sqrt{r(S)})^{-1}$, i.e. because the wavenumber is independent of the axial coordinate the amplitude variation is proportional to $(\sqrt{r(S)})^{-1}$ - exactly as in the shell of revolution, c.f. equation (19).

The coupling between the axial and breathing mode is reserved for the shell of revolution, and does not occur for the rod and beam, although in the case of $v=0$ these two waves uncouple and thus a counterpart in the simpler theories can be found. In this case the wavenumber of the axial mode, in the shell of revolution, may be determined from $M_{11}=0$ while the amplitude is determined by the solvability condition $\alpha_{0}(S) \overline{f_{1}}=0 \Rightarrow \overline{f_{1}}=0$. Thus the wavenumber is $k_{a}(S)=\omega$ while the amplitude is $\alpha_{a}(S) \propto(\sqrt{r(S)})^{-1}$. The same result is found if the governing equation for the axial wave in a rod with varying cross section is non-dimensionalised and solved by means of the WKB method, again see [11].

Finally, the counterpart of the breathing mode in the beam and rod theory might not be as obvious as for the torsion and axial mode. Nevertheless it is well-known in the cylindrical shell theory that this mode behaves in an analogous manner to the Euler-Bernoulli beam attached to a Winkler foundation, although to take the varying radius into account a few modifications, which are presented in appendix B, are needed. Using this model and solving it with a similar WKB-solution as before, the same results are found as if $M_{33}=0$ and $\overline{f_{3}}=0$, i.e. $k_{b}(S)=\left(\frac{12}{\delta^{2}}\left(\omega^{2}-r(S)^{-2}\right)\right)^{1 / 4}$ and $\gamma_{b}(S)=\left(k(S)^{3 / 2} \sqrt{r(S)}\right)^{-1}$.

\subsubsection{When $m>0$ - Bending and Higher-order Modes}

When the circumferential wavenumber is $m>0$ the matrix $\mathbf{M}$ remains fully populated. Although the solution algorithm follows what has been described above the functions $Q(S)$ and $P(S)$ become so complicated that the integration of equation (14) can hardly be done analytically. Instead this step can be done numerically. However, if the integration is to be done numerically, it also becomes necessary to evaluate $k^{\prime}(S)$, see e.g. equation (17) and (18). In this general case the dispersion relation turns out to be an eight order polynomial. But as before it only has even powers of the wavenumber and can thus be solved as an implicit fourth order polynomial. Again these analytical roots become very cumbersome, and therefore also in this case it becomes simpler to solve the dispersion relation numerically instead. Nevertheless $k^{\prime}(S)$ can be found explicitly by implicit differentiation of the eight order dispersion relation, and then isolate $k^{\prime}(S)$ analytically. Fortunately this method provides a rather simple expression.

\subsection{Energy Flux}

Because free undamped waves are under consideration in this paper, the validation of the derived WKB solution relies on the statement that the time-averaged power, flowing through the shell of revolution, is conserved. Nevertheless it should be emphasised that because the solution is a leading order approximation the conservation of power can only be expected to hold to leading order. The time-averaged power flow can be assessed by considering a section of the shell of revolution like the one bounded by $s_{1}$ and $s_{2}$ in figure 1 . For the initial statement to be true, the time-averaged power passing the boundary at $s_{1}$ must be equal to the time-averaged power passing at $s_{2}$. The power passing the boundaries at $s_{1}$ and $s_{2}$ is associated with the shells oscillations along the $u$-, $v$-, and $w$-axis as well as around the $v$-axis. In fact the oscillations along the $w$-axis and around the $v$-axis are coupled and can together be considered as local bending of the shell wall. When a point along one of the boundaries is displaced or rotated the shell structure will respond with a reaction force or a reaction moment, known as generalised forces. By properly multiplying the displacements and generalised forces the time-averaged power passing the boundary may be calculated as, cf. [12], 


$$
\begin{aligned}
& \Pi_{u}^{*}=\frac{1}{2} \operatorname{Re}\left\{\Phi_{u, 0}^{*} \overline{\dot{u}_{0}^{*}}\right\}=-\frac{\omega c^{*}}{2} \operatorname{Re}\left\{i \Phi_{u, 0}^{*} \overline{\tilde{u}_{0}}\right\} \\
& \Pi_{v}^{*}=\frac{1}{2} \operatorname{Re}\left\{\Phi_{v, 0}^{*} \overline{\dot{v}_{0}^{*}}\right\}=-\frac{\omega c^{*}}{2} \operatorname{Re}\left\{i \Phi_{v, 0}^{*} \overline{\tilde{v}_{0}}\right\} \\
& \Pi_{w}^{*}=\frac{1}{2} \operatorname{Re}\left\{\Phi_{w, 0}^{*} \overline{\dot{w}_{0}^{*}}\right\}=-\frac{\omega c^{*}}{2} \operatorname{Re}\left\{i \Phi_{w, 0}^{*} \overline{\tilde{w}_{0}}\right\} \\
& \Pi_{w^{\prime}}^{*}=\frac{1}{2} \operatorname{Re}\left\{\Phi_{w^{\prime}, 0}^{*} \overline{\partial \dot{w}_{0}^{*}}\right\}=-\frac{\omega c^{*}}{2} \operatorname{Re}\left\{i \Phi_{w^{\prime}, 0}^{*} \overline{\partial \tilde{w}_{0}} \frac{\partial S}{\partial S}\right\} \\
& \Pi_{\text {bending }}^{*}=\Pi_{w}^{*}+\Pi_{w^{\prime}}^{*} \\
& \Pi_{\text {total }}^{*}=\Pi_{u, 0}^{*}+\Pi_{v, 0}^{*}+\Pi_{\text {bending }}^{*},
\end{aligned}
$$

where $\Phi_{i}$ is the generalised force associated with $i$-motion and $\bar{x}$ is the complex conjugated of $x$. The generalised forces for the special case of a uniform cylinder are derived in the paper by [13], though for the general shell of revolution they have not been found available in the scientific literature. Thus these will be derived from scratch by means of Hamilton's principle. Because the generalised forces will be derived independently and then multiplied by the WKB solution, in accordance with equations (27), the behaviour of the obtained power flow will serve as a demonstration of the validity of the calculations. Hence the derivation of the generalised forces for the shell of revolution will be presented in some detail in the following, and will start out with the definition of Hamilton's principle:

$$
\delta\left(\int_{t_{1}^{*}}^{t_{2}^{*}}\left(T^{*}-U^{*}\right) d t^{*}\right)=0,
$$

where $T^{*}$ is the kinetic energy of the motion of the shell and $U^{*}$ is the potential energy of deformation of the shell. This states that the body forces due to the deformation of the shell will over time be balanced by the inertia of the shell's own mass. Because the generalised forces are a result of body forces then it is possible to extract them from the first variation of the potential energy. Thus, from [8] the potential energy of the section bounded by $s_{1}$ and $s_{2}$ is:

$$
\begin{aligned}
U^{*}= & \frac{E^{*} h^{*}}{2\left(1-v^{2}\right)} \int_{0}^{2 \pi} \int_{s_{1}}^{s_{2}}\left(\left(\varepsilon_{s}+\varepsilon_{\theta}\right)^{2}-2(1-v)\left(\varepsilon_{s} \varepsilon_{\theta}-\frac{\gamma^{2}}{4}\right)\right) A_{s}^{*} A_{\theta}^{*} d s d \theta+ \\
& +\frac{E^{*} h^{* 3}}{24\left(1-v^{2}\right)} \int_{0}^{2 \pi} \int_{s_{1}}^{s_{2}}\left(\left(\kappa_{s}^{*}+\kappa_{\theta}^{*}\right)^{2}-2(1-v)\left(\kappa_{s}^{*} \kappa_{\theta}^{*}-\tau^{* 2}\right)\right) A_{s}^{*} A_{\theta}^{*} d s d \theta .
\end{aligned}
$$

Taking the first variation with respect to deformations gives

$$
\begin{aligned}
\delta\left(U^{*}\right)= & \frac{E^{*} h^{*}}{2\left(1-v^{2}\right)} \int_{0}^{2 \pi} \int_{s_{1}}^{s_{2}}\left(2\left(\varepsilon_{s} \delta\left(\varepsilon_{s}\right)+\varepsilon_{\theta} \delta\left(\varepsilon_{\theta}\right)\right)+2 v\left(\varepsilon_{\theta} \delta\left(\varepsilon_{s}\right)+\varepsilon_{s} \delta\left(\varepsilon_{\theta}\right)\right)+(1-v) \gamma \delta(\gamma)\right) A_{s}^{*} A_{\theta}^{*} d s d \theta+ \\
& +\frac{E^{*} h^{* 3}}{24\left(1-v^{2}\right)} \int_{0}^{2 \pi} \int_{s_{1}}^{s_{2}}\left(2\left(\kappa_{s}^{*} \delta\left(\kappa_{s}^{*}\right)+\kappa_{\theta}^{*} \delta\left(\kappa_{\theta}^{*}\right)\right)+2 v\left(\kappa_{\theta}^{*} \delta\left(\kappa_{s}^{*}\right)+\kappa_{s}^{*} \delta\left(\kappa_{\theta}^{*}\right)\right)+4(1-v) \tau^{*} \delta\left(\tau^{*}\right)\right) A_{s}^{*} A_{\theta}^{*} d s d \theta .
\end{aligned}
$$

Actually, the constitutive relations in (40) are in Novozhilov's textbook extracted by collecting $\delta\left(\varepsilon_{s}\right)$-terms, $\delta\left(\varepsilon_{\theta}\right)$ terms, $\delta\left(\kappa_{s}\right)$-terms, etc. from this expression. Nevertheless, the aim is here to relate the shell reactions to displacements, and not just to deformations. Thus the variation is taken a step further by substituting the displacementdeformation relations from (39) and taking the first variation with respect to displacements. For simplicity, in the next 
step only the integration of first term will be presented. So, by means of integration by parts over the section bounded by $s_{1}$ and $s_{2}$ the following is obtained:

$$
\begin{aligned}
\int_{0}^{2 \pi} \int_{s_{1}}^{s_{2}} \varepsilon_{s} \delta\left(\varepsilon_{s}\right) A_{s}^{*} A_{\theta}^{*} d s d \theta & =\int_{0}^{2 \pi} \int_{s_{1}}^{s_{2}} \varepsilon_{s} \delta\left(\frac{1}{A_{s}^{*}} \frac{\partial u^{*}}{\partial s}+\frac{1}{R_{s}^{*}} w^{*}\right) A_{s}^{*} A_{\theta}^{*} d s d \theta= \\
& =\int_{0}^{2 \pi} \int_{s_{1}}^{s_{2}} \varepsilon_{s}\left(A_{\theta}^{*} \delta\left(\frac{\partial u^{*}}{\partial s}\right)+\frac{A_{s}^{*} A_{\theta}^{*}}{R_{s}^{*}} \delta\left(w^{*}\right)\right) d s d \theta= \\
& =\int_{0}^{2 \pi}\left(\left[A_{\theta}^{*} \varepsilon_{s} \delta\left(u^{*}\right)\right]_{s_{1}}^{s_{2}}-\int_{s_{1}}^{s_{2}} \frac{\partial}{\partial s}\left(A_{\theta}^{*} \varepsilon_{s}\right) \delta\left(u^{*}\right) d s+\int_{s_{1}}^{s_{2}} \frac{A_{s}^{*} A_{\theta}^{*}}{R_{s}^{*}} \delta\left(w^{*}\right) d s\right) d \theta
\end{aligned}
$$

At this stage the bracket evaluated at $s_{1}$ and $s_{2}$ will vanish as $\delta\left(u^{*}\right)$ is zero at the boundaries by definition. However the quantity $A_{\theta}^{*} \varepsilon_{s}$, in front of $\delta\left(u^{*}\right)$, may indeed not necessarily be zero. Because $A_{\theta}^{*} \varepsilon_{s}$ is multiplied with a displacement and the product in the end is potential energy, then $A_{\theta}^{*} \varepsilon_{s}$ multiplied with the constant in front of the integral can be regarded as a force. By writing out the integration in (30) term by term in this way, and collecting the quantities multiplied with, respectively, $\delta\left(u^{*}\right), \delta\left(v^{*}\right), \delta\left(w^{*}\right)$, and $\delta\left(\frac{\partial w^{*}}{\partial s}\right)$ from the brackets evaluated at $s_{1}$ and $s_{2}$, the generalised forces are obtained:

$$
\begin{aligned}
& \Phi_{u}^{*}=\frac{E^{*} h^{*}}{1-v^{2}} A_{\theta}^{*}\left(\varepsilon_{s}+v \varepsilon_{\theta}+\frac{h^{* 2}}{12 R_{s}^{*}}\left(\kappa_{s}^{*}+v \kappa_{\theta}^{*}\right)\right)=A_{\theta}^{*}\left(T_{s}^{*}+\frac{M_{s}^{*}}{R_{s}^{*}}\right) \\
& \Phi_{v}^{*}=\frac{E^{*} h^{*}}{2(1+v)} A_{\theta}^{*}\left(\gamma+\frac{h^{* 2}}{6 R_{\theta}^{*}} \tau^{*}\right)=A_{\theta}^{*} T_{s \theta}^{*} \\
& \Phi_{w}^{*}=\frac{E^{*} h^{* 3}}{12\left(1-v^{2}\right)} \frac{1}{A_{s}^{*}}\left(\frac{\partial}{\partial s}\left(A_{\theta}^{*}\left(\kappa_{s}^{*}+v \kappa_{\theta}^{*}\right)\right)-\frac{\partial A_{\theta}^{*}}{\partial s}\left(\kappa_{\theta}^{*}+v \kappa_{s}^{*}\right)+2(1-v) A_{s}^{*} \frac{\partial \tau^{*}}{\partial \theta}\right)=A_{\theta}^{*} N_{s}^{*}+\frac{\partial M_{s \theta}^{*}}{\partial \theta} \\
& \Phi_{w}^{\prime *}=-\frac{E^{*} h^{* 3}}{12\left(1-v^{2}\right)} \frac{A_{\theta}^{*}}{A_{s}^{*}}\left(\kappa_{s}^{*}+v \kappa_{\theta}^{*}\right)=-\frac{A_{\theta}^{*}}{A_{s}^{*}} M_{s}^{*},
\end{aligned}
$$

where the constitutive relations in (40) are used to write the generalised forces in their most compact form. These generalised forces hold for any shell of revolution. Now, in order to calculate the power flow associated with the displacements determined by the WKB solution the expressions in (32) are manipulated in the following way: Firstly, the displacement-deformation relations from equations (39), the Lamé parameters, and the radii of curvature from (5) are substituted into the expressions. After that, the general WKB solution in (1) combined with (7) is substituted and the generalised forces are expanded after the series $\Phi_{i, 0}^{*}+\varepsilon \Phi_{i, 1}^{*}+\varepsilon^{2} \Phi_{i, 2}^{*}+\ldots$. By finally collecting $O(1)$-terms the leading order generalised forces for the shell of revolution with slowly varying radius become: 


$$
\begin{aligned}
\Phi_{u, 0}^{*} & =E^{*} r_{0}^{* 2} \frac{\delta}{1-v^{2}}\left(i r(S) k(S) \tilde{u}_{0}+i m v \tilde{v}_{0}-v \tilde{w}_{0}\right) \\
\Phi_{v, 0}^{*} & =E^{*} r_{0}^{* 2} \frac{\delta}{2(1+v)} i\left(m \tilde{u}_{0}+r(S) k(S) \tilde{v}_{0}+\frac{i \delta^{2} m k(S)}{6 r(S)} \tilde{w}_{0}\right) \\
\Phi_{w, 0}^{*} & =-E^{*} r_{0}^{* 2} \frac{\delta^{3}}{12\left(1-v^{2}\right) r(S)}\left((2-v)\left(m k(S) \tilde{v}_{0}+i m^{2} k(S) \tilde{w}_{0}\right)+i r(S)^{2} k(S)^{3} \tilde{w}_{0}\right) \\
\Phi_{w^{\prime}, 0}^{*} & =E^{*} r_{0}^{* 2} \frac{\delta^{3}}{12\left(1-v^{2}\right) r(S)}\left(i v m \tilde{v}_{0}-r(S)^{2} k(S)^{2} \tilde{w}_{0}-v m^{2} \tilde{w}_{0}\right) .
\end{aligned}
$$

As can be seen $\Phi_{i, 0}$ are in all four cases a linear combination of the components of $\tilde{\mathbf{u}}_{0}$. Thus the exponential functions of $s$ and $\theta$ can be taken outside of a common bracket which will then vanish, when multiplied with its complex conjugated in (27). This means that $\tilde{\mathbf{u}}_{0}$ can just be replaced by $\boldsymbol{\alpha}_{0}$ when the expressions above are used in (27).

\subsubsection{Power Flow when $m=0$ - The Axial and Torsional Wave Mode}

In the case of torsional wave motion the power is only carried by circumferential displacement. Thus only equations (27b) and (33b) are used. By substituting the WKB approximation of the torsion amplitude from equation (19) the associated power flow is

$$
\Pi_{t}^{*}=C_{t}^{2} E^{*} c^{*} r_{0}^{* 2} \frac{\delta \omega}{6(1+v)}
$$

which indeed is independent of the axial coordinate.

Likewise the power due to the axial/breathing mode can be calculated. As this mode consists of axial and normal displacement, as well as rotation around the $v$-axis, the total power flow can be determined as

$$
\Pi_{a}^{*}=C_{a}^{2} E^{*} c^{*} r_{0}^{* 2} \frac{\delta \omega}{6\left(1-v^{2}\right) v^{2}}
$$

which is also independent of the axial coordinate.

\subsubsection{Power Flow when $m>0$}

The WKB solution when $m>0$ has, as explained previously, only been found numerically. This means that the power flow must be calculated numerically by using a specific radius variation of some predefined shell of revolution and then stepping through a sequence of $S$-values to check whether the power is conserved. However this will not be presented here. Moreover the numerical implementation of the WKB solution can be validated by replicating the analytical WKB-solution for $m=0$ for some characteristic predefined shells of revolution.

\subsection{Energy Distribution}

While it may be claimed that the power flow must be conserved along the shell this statement says nothing about how the mechanical energy, which is the sum of kinetic and potential energy, may be distributed along the shell. However, it can be shown mathematically that at each location along the waveguide the time-averaged kinetic and potential energy are identical, see [14]. The time-average is obtained by integrating over at least one full wave cycle. Thus, by introducing the notation $\widehat{x}=\frac{1}{t_{p}} \int_{0}^{t_{p}} x d t$ where $t_{p}$ is the time required to complete one cycle, it can be stated that $\widehat{T}^{*}=\widehat{U}^{*}$. However, both the kinetic and potential energies are obtained by integrating their point-wise contribution 
over the shell, i.e. $T^{*}=\int_{0}^{2 \pi} \int_{s_{1}}^{s_{2}} \xi^{*} d s d \theta$ and $U^{*}=\int_{0}^{2 \pi} \int_{s_{1}}^{s_{2}} v^{*} d s d \theta$, and thus $\widehat{\xi}^{*}=\widehat{v}^{*}$. It can be found that the time-average of the point-wise kinetic and potential are:

$$
\begin{aligned}
\widehat{\xi}^{*}= & \frac{\rho^{*} h^{*}}{4} \omega^{2}\left(\tilde{u}_{0} \overline{\tilde{u}_{0}}+\tilde{v}_{0} \overline{\tilde{v}_{0}}+\tilde{w}_{0} \overline{\tilde{w}_{0}}\right) \\
\widehat{v}^{*}= & \frac{E^{*} h^{*}}{2\left(1-v^{2}\right)}\left(\frac{1}{2}\left(\operatorname{Re}\left\{\widehat{\varepsilon}_{s}+\widehat{\varepsilon}_{\theta}\right\}^{2}+\operatorname{Im}\left\{\widehat{\varepsilon}_{s}+\widehat{\varepsilon}_{\theta}\right\}^{2}\right)-(1-v)\left(\operatorname{Re}\left\{\widehat{\varepsilon_{s}} \widehat{\widehat{\varepsilon}_{\theta}}\right\}-\frac{1}{4} \operatorname{Re}\{\widehat{\gamma \gamma}\}\right)\right)+ \\
& +\frac{E^{*} h^{* 3}}{24\left(1-v^{2}\right)}\left(\frac{1}{2}\left(\operatorname{Re}\left\{\widehat{\kappa_{s}^{*}}+\widehat{\kappa_{\theta}^{*}}\right\}^{2}+\operatorname{Im}\left\{\widehat{\kappa_{s}^{*}}+\widehat{\kappa_{\theta}^{*}}\right\}^{2}\right)-(1-v)\left(\operatorname{Re}\left\{\widehat{\kappa_{s}^{*}} \widehat{\kappa_{\theta}^{*}}\right\}-\operatorname{Re}\left\{\widehat{\tau^{*}} \widehat{\tau^{*}}\right\}\right)\right) .
\end{aligned}
$$

Thus, despite the fact that the structure of these two expressions is rather different, they must still be equal. In this way the comparison between the time-averaged kinetic and potential energy is yet another sanity-check of the calculations.

\section{Results and Discussions}

To demonstrate the capabilities of the derived model, the wave guide properties of the two shells of revolution illustrated in figure 2 will be examined. The radius function is in each case expressed by:

$$
\text { (a): } r(S)=b+a \tanh (S) \quad \text { (b): } r(S)=S+b,
$$

where the coefficients are given in the figure captions. Shell (a) is interesting because it is composed of two cylindrical shells which are connected by a smooth transition. The smooth transition contains sections of double curved surfaces with, respectively, positive and negative Gaussian curvature ${ }^{2}$. Such features are seen in applications like jet engines, wind instruments, piping systems, etc. Typically a positive Gaussian curvature has a stiffening effect on the shell as opposed to the negative Gaussian curvature. However, as it is assumed that the waves locally see the shell as a uniform cylinder, i.e. with a Gaussian curvature of zero, this stiffening effect is expected only to play a minor if any role at all. Thus the important characteristic of shell (a) is rather that it simply represents a differentiable transition between two uniform cylinders. To back up this claim shell (b) is considered. This conical shell is the simplest perturbation from the uniform cylinder and obviously has a linearly changing radius and a Gaussian curvature of zero at any point of its surface.

In what follows, the primary focus will be on the axial/breathing mode $(m=0)$. Even though this is just a fundamental and rather simple mode it is complicated enough to reveal a quite sophisticated behaviour as the wave is travelling along the shell of revolution. For shell (a), the dispersion relation, modal unit vector, amplitude, and energy distribution as functions of frequency and location will be considered. Then, for shell (b), the dispersion relation, modal unit vector, and amplitude will be treated in the same way. Finally, as an example of a higher order, though still fundamental, mode the dispersion relation, modal unit vector, and amplitude of the waves related to $m=1$, which among others include the global bending mode, will be presented.

\subsection{Dispersion Relation - Shell (a)}

As described previously the dispersion relation of the axial/breathing mode is obtained from the $O(1)$-system, that is equation (8a), by taking $M_{11} M_{33}-M_{13}^{2}=0$ and $m=0$ and substituting the radius function for shell (a). Hence, the results in this subsection, as well as the subsequent subsection concerning the modal unit vector, uncover how the dispersion relation and modal unit vector of a classical uniform cylindrical shell depends on the local value of the radius function.

\footnotetext{
${ }^{2}$ The Gaussian curvature is defined as the product of the two principal curvatures of a surface, i.e. in this case $\frac{1}{R_{s}} \frac{1}{R_{\theta}}$.
} 


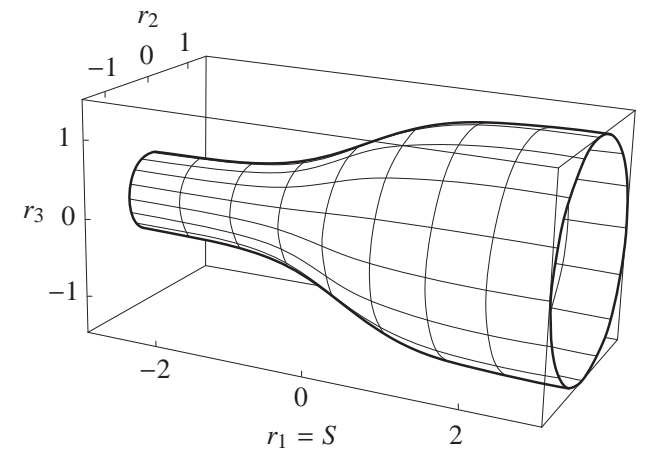

(a): $b=1, a=0.5$

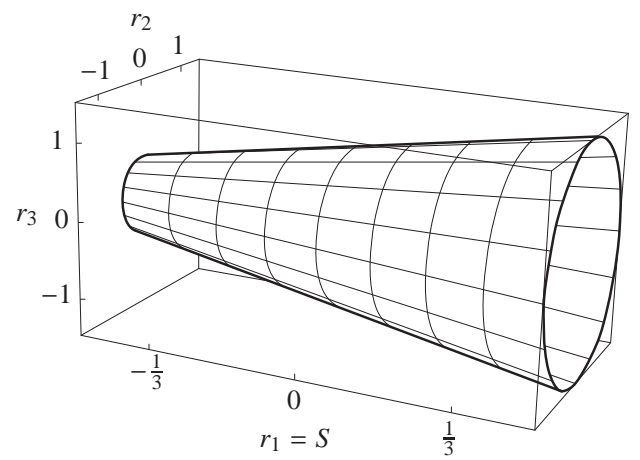

(b): $b=1$

Figure 2: Shell (a) represents a smooth transition from one constant radius to another, while shell (b) is a conical shell

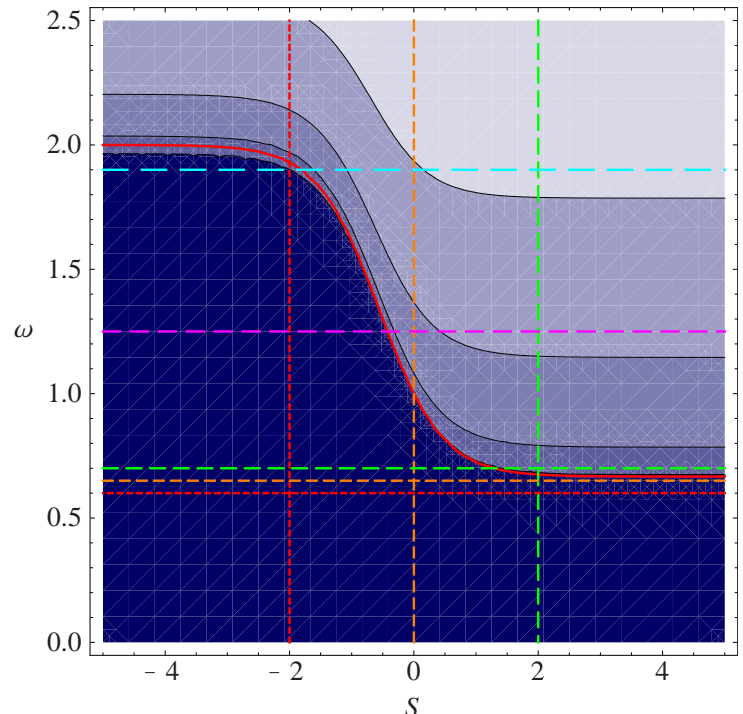

(a)

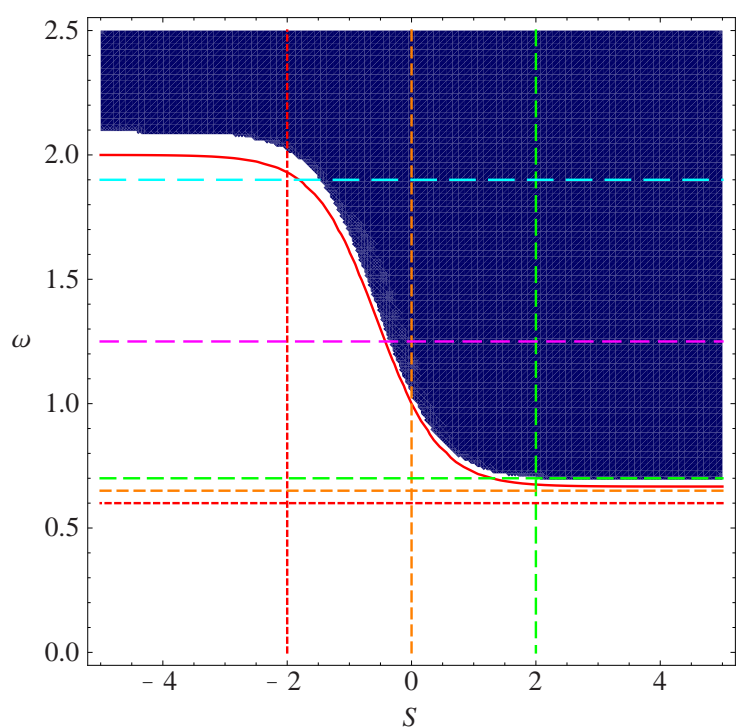

(b)

Figure 3: Contour plot of the wavenumber of (a) the 1st axial/breathing wave and (b) the 2nd axial/breathing wave.

From the dispersion relation of the axial/breathing mode, two travelling waves are predicted. The contour plot of the 1st wave is illustrated in figure 3(a) while the contour plot of the 2nd wave is illustrated in (b). In both contour plots the location along the shell, in terms of the slow coordinate, is running along the horizontal axis while the frequency is running along the vertical axis. The shading of the contour levels is such that a lighter blue corresponds to a higher wavenumber and vice versa. However, it should be remarked that pure white indicates that the wave is not travelling, but is evanescent, at that location in the contour plot. Thus by imagining plot (a) on top of plot (b) it is seen that the wavenumber of the 1 st wave increases dramatically as soon as the 2 nd wave cuts on. In this way the contour plots give a broad overview of how the dispersion relation qualitatively depends on the location and frequency. To quantify the contour levels the plots in figure 4 are presented. These plots contain both the 1st and 2nd waves, but for fixed locations in (a) and fixed frequencies in (b). The fixed locations and frequencies are also indicated in the contour plots by the coloured dashed lines. The plots in figure 4 demonstrate more precisely how the wavenumbers of the 1 st and 2nd waves do not cross each other, but instead the 1 st wave veers off somewhat before the 2 nd wave cuts on. This veering effect is already well known, see e.g. [15], and is a direct consequence of the two waves being coupled through the Poisson effect, even though they still are linearly independent. However, these results also demonstrate how the 


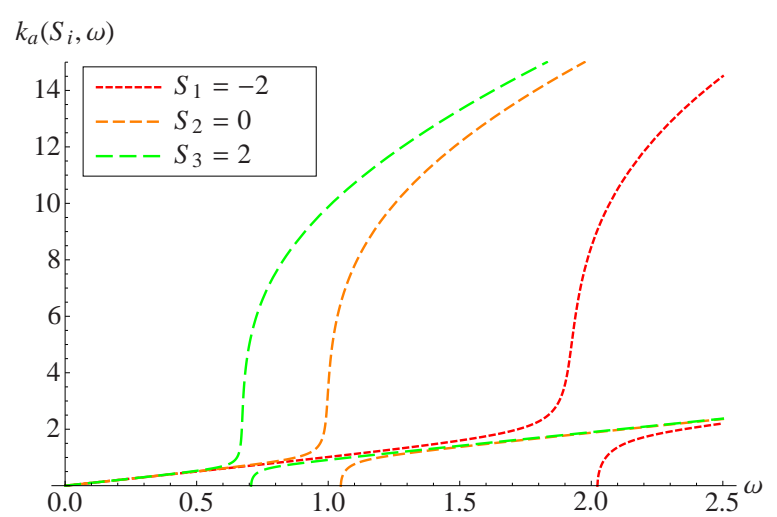

(a)

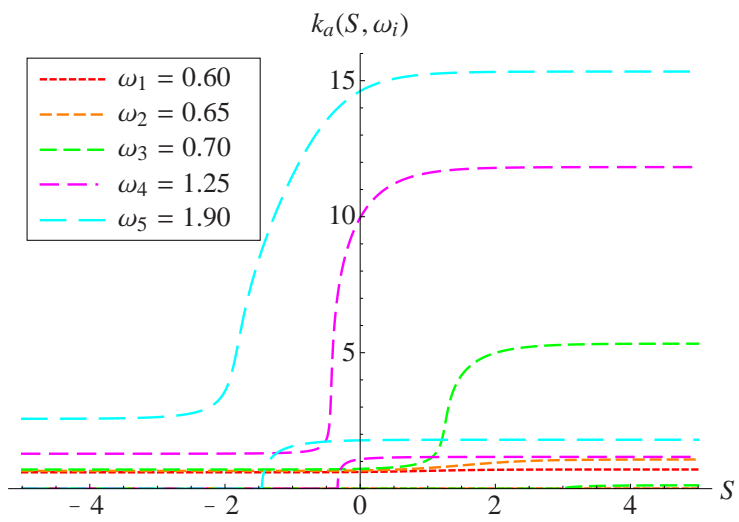

(b)

Figure 4: Along the fixed frequencies and locations indicated by the dashed lines in figure 3, the wavenumber is plotted as function of (a) the frequency and (b) the slow coordinate.

cut-on of the 2nd wave in this case can be affected by the changing radius. Hence figure 3(b) and 4(b) demonstrate that over a rather broad range of frequencies the 2 nd wave only becomes travelling when the radius of the shell becomes large enough and, in this particular example, below a frequency of $\omega \simeq 0.65$ the 2 nd wave does not becomes travelling at all. In fact, the relationship between the slow coordinate and the frequency, that determines where the veering and cut-on of 2 nd wave occurs, is roughly indicated by the red s-shaped curve in the contour plots. This curve is exactly the so-called ring-frequency as a function of the slow coordinate. This will be further discussed in subsection 3.2.1.

\subsection{Modal Unit Vector - Shell (a)}

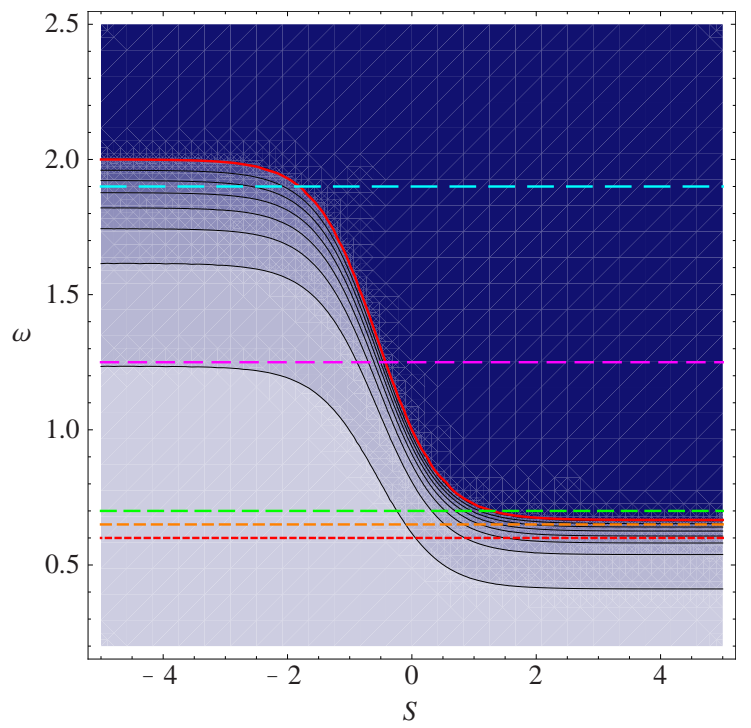

(a)

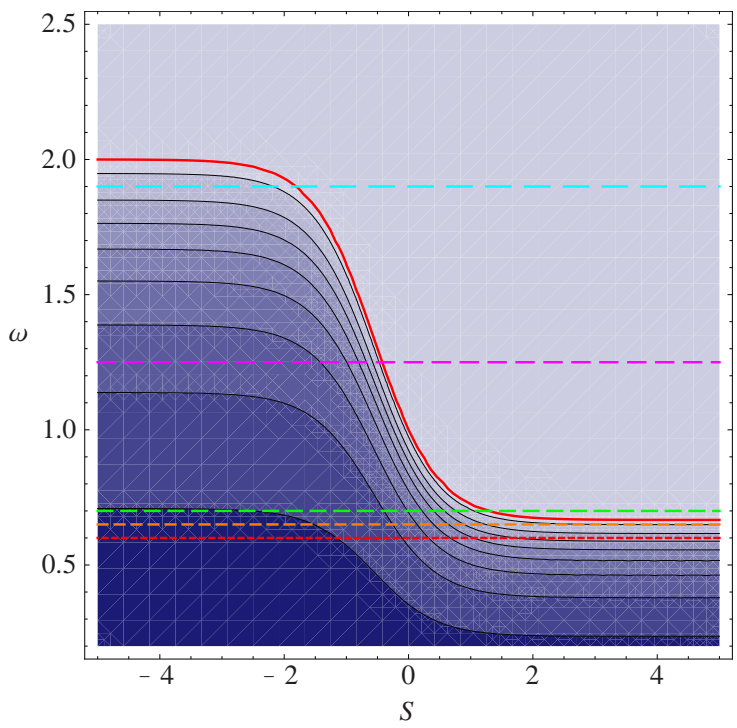

(b)

Figure 5: Contour plots of (a) the first component of the modal unit vector related to the 1 st axial/breathing wave, i.e. $\frac{\left|\alpha_{a}(S, \omega)\right|}{\left|\alpha_{a}(S, \omega)\right|}$ and (b) the third component, i.e. $\frac{\left|\gamma_{a}(S, \omega)\right|}{\left|\boldsymbol{\alpha}_{a}(S, \omega)\right|}$.

To gain more insight about how the 1st and the 2nd waves behave it is, as described by [15], useful to plot the components of the modal unit vector, i.e. the modal coefficients, see equations (20), (23), and (24). Thus, as the axial/breathing wave consists of axial and normal motion, the contour plot of the axial component of the modal unit vector related to the 1st wave is illustrated in figure 5(a) while the contour plot of the normal component is illustrated 


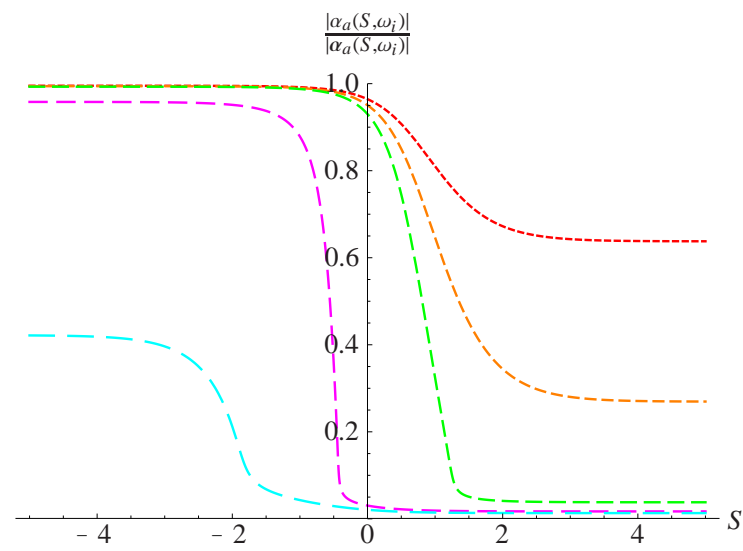

(a)

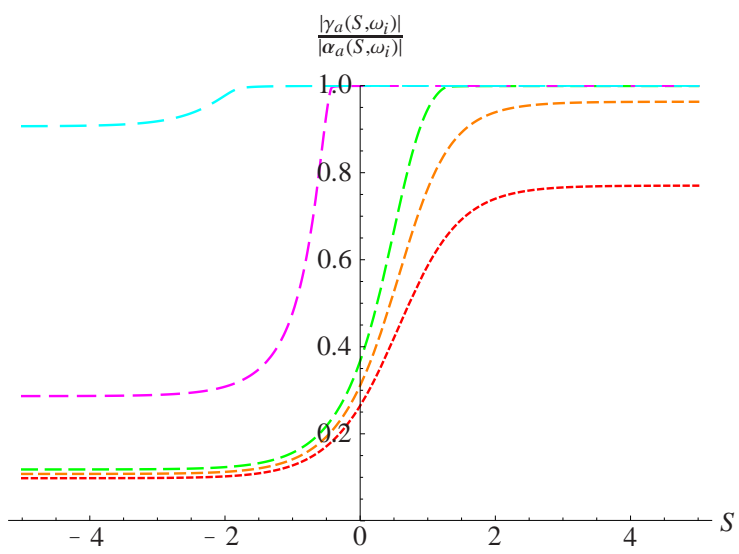

(b)

Figure 6: Along the fixed frequencies indicated by the dashed lines in figure 5, the components of the modal unit vector are plotted as function of the slow coordinate.

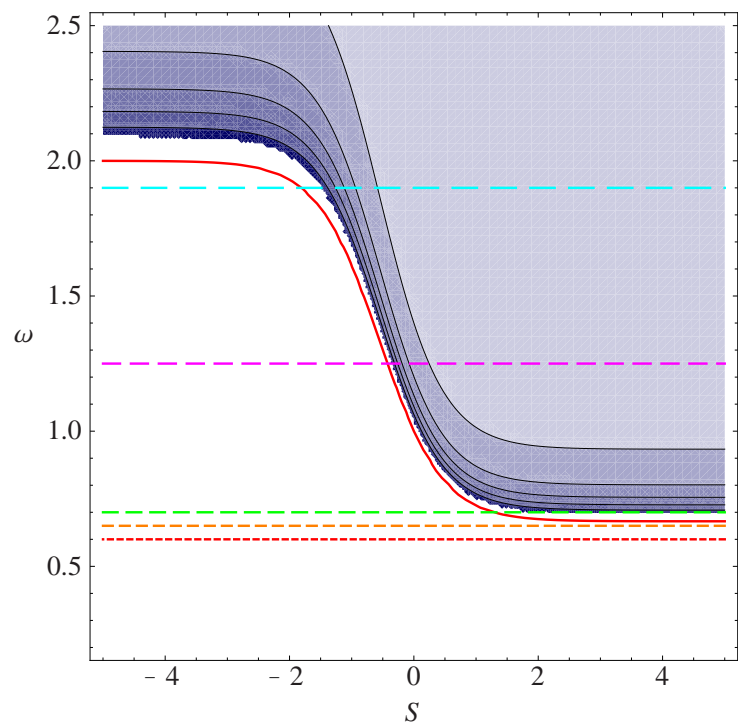

(a)

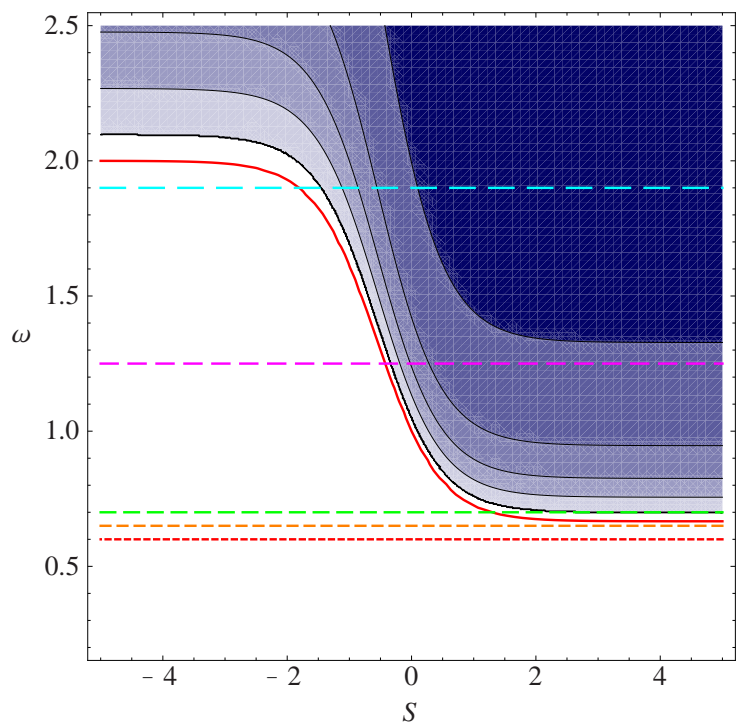

(b)

Figure 7: Contour plots of (a) the first component of the modal unit vector related to the 2 nd axial/breathing wave, i.e. $\frac{\left|\alpha_{a}(S, \omega)\right|}{\left|\alpha_{a}(S, \omega)\right|}$ and (b) the third component, i.e. $\frac{\left|\gamma_{a}(S, \omega)\right|}{\alpha_{a}(S, \omega) \mid}$. In the white area the 2 nd axial/breathing wave is evanescent and thus not travelling.

in 5(b). The axial and normal component, at the fixed frequencies indicated in these contour plots, are plotted in figure 6(a) and 6(b). As marked on top of the vertical axis the modal coefficients are normalised by the norm of $\alpha_{a}$ which by definition is: $\left|\alpha_{a}\right|=\sqrt{\left|\alpha_{a}\right|^{2}+\left|\beta_{a}\right|^{2}+\left|\gamma_{a}\right|^{2}}$. Similarly, the modal coefficients of the 2nd wave are illustrated in figure 7 and figure 8. Again it should be remarked that the purely white area of the contour plot is where the 2 nd wave is not yet travelling. However, this does not mean that the 2 nd wave does not give rise to displacements before cut-on. But as mentioned earlier, the wave is evanescent until cut-on, and only the travelling part of the wave is considered here.

Now, in relation to the red s-shaped curve in figure 5, the contour plots show that the modal unit vector for the 1 st wave changes rapidly somewhat before the red curve. The modal unit vector of the 2 nd wave, on the other hand, changes rapidly as soon as the wave cuts on a bit after the red curve, and then afterwards attains a constant level. Figure 6 also shows that the 1st wave is dominated by axial motion before the veering, and is dominated by normal motion after the veering. Exactly the opposite is true for the 2 nd wave which consists purely of normal motion right at cut-on, but then rapidly shifts towards being dominated by axial motion. Together with the dispersion diagrams from the previous subsection this demonstrates how the 1 st and 2 nd waves convert into one another during the veering and 


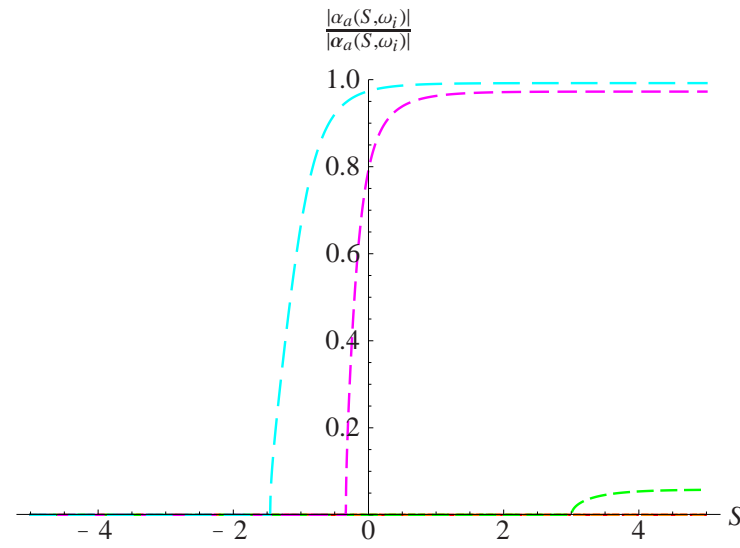

(a)

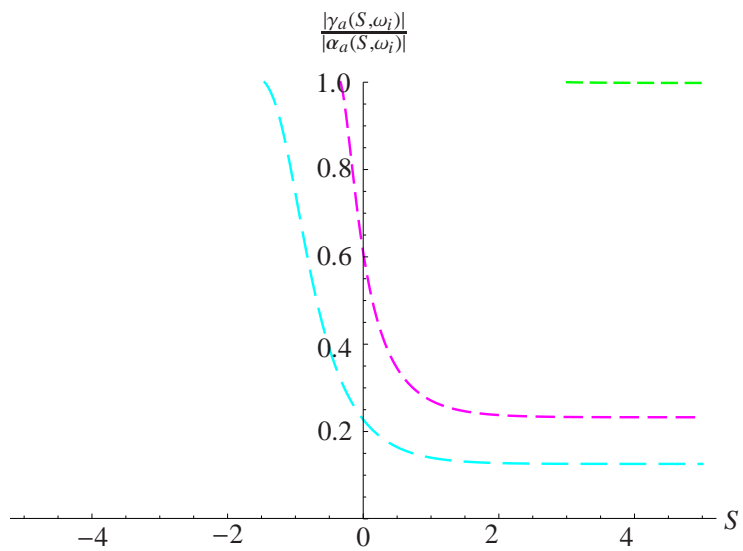

(b)

Figure 8: Along the fixed frequencies indicated by the dashed lines in figure 7, the components of the modal unit vector are plotted as function of the slow coordinate.

continue after the veering as each other's extension. In other words, before the veering the 1st wave is an axial wave, which due to the Poisson's coupling is forcing the breathing wave to be present. In that way the breathing wave is a slave of the axial wave, as long as the veering has not occurred yet. Then, after the veering, the 1st wave has converted into a breathing wave, which then again due to the Poisson's coupling is now forcing the axial wave to be present. Thus the axial wave becomes a slave of the breathing wave. In fact, this interpretation is backed up by the simpler rod and beam models and will be discussed further in the following subsection.

\subsubsection{Dispersion Relation and Modal Vectors in Relation to Fundamental Rod, Beam, and Cylindrical Shell Theory}

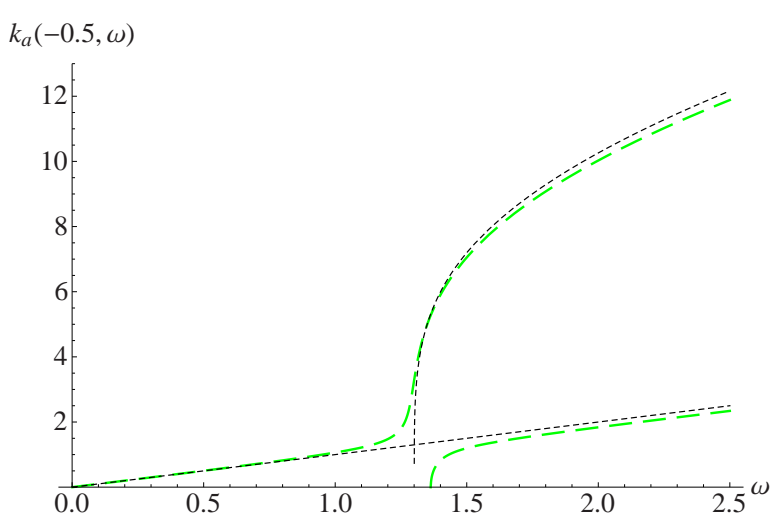

(a)

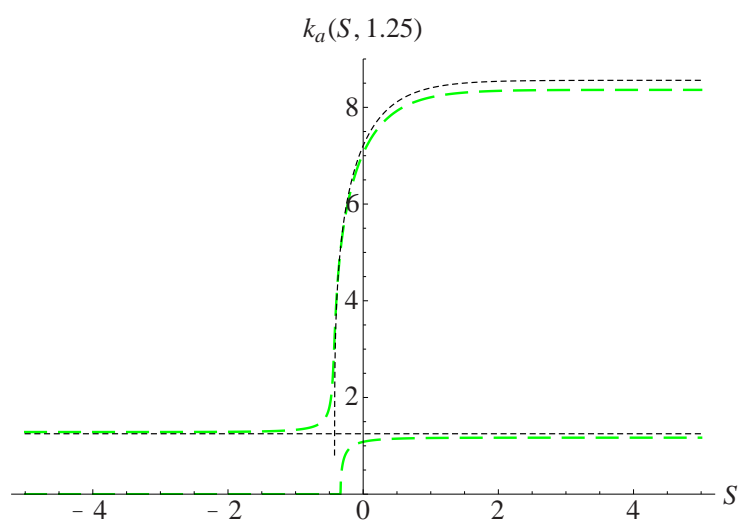

(b)

Figure 9: At $S=-0.5$ the dispersion relation as function of the frequency is plotted in (a) whereas in (b) the dispersion relation is plotted as function of location at $\omega=1.25$. The dashed, thin, and straight curve is the dispersion relation for the axial wave in a rod while the dashed, thin, and non-straight curve is the dispersion relation for the breathing wave modelled as a bending beam on a Winkler foundation.

In subsection 2.1.4 the axial wave in a rod, the bending wave in a beam on a Winkler foundation, and the decoupled axial/breathing wave in the shell of revolution were found to be pairwise identical. The axial/breathing wave was decoupled by equating Poisson's ratio to zero in the $O(1)$-system and then separately determining the wavenumber and amplitude of the two waves. In spite of the continuous nature of Poisson's coupling, the coupled axial/breathing wave also has a close connection to the decoupled axial and breathing wave. Hence the dispersion relation for the axial wave in a rod and the bending wave in a beam on a Winkler foundation is compared to the coupled axial/breathing wave in figure 9. This figure clearly shows how the 1 st wave before the veering, and the 2 nd wave after the veering, asymptotically approach the dispersion relation of the axial wave in a rod. Likewise, the 2nd wave cuts on right after the cut-on of the bending wave, though because of the veering the 1st wave takes over to asymptotically approach this 
branch. Moreover, the modal vector of the decoupled axial wave obviously only contains an axial component, while the modal vector of the decoupled breathing wave only contains a normal component.

It was previously mentioned that the red s-shaped curve, which in the contour plots above indicates the location of the veering, specifies the relation between the frequency and location of the ring-frequency. For the decoupled breathing wave this frequency is identical to its cut-on frequency and matches the eigenfrequency of a closed ring which is undergoing breathing motion. Actually, this eigenfrequency is modelled by omitting the bending term from the model of the bending beam on a Winkler foundation, c.f. appendix B, and solving equation (45) for $\omega$. From this, the ring-frequency is given by $\omega_{r}=r(S)^{-1}$. Hence, because of the veering of the coupled axial/breathing wave, the cut-on of the 2nd wave will happen shortly after the ring-frequency. Besides this, at the location where the radius of the shell and the frequency of the wave match the ring-frequency, the local dynamic stiffness towards breathing motion will be decreased and thus potentially creating localised resonance.

\subsection{Amplitude - Shell (a)}

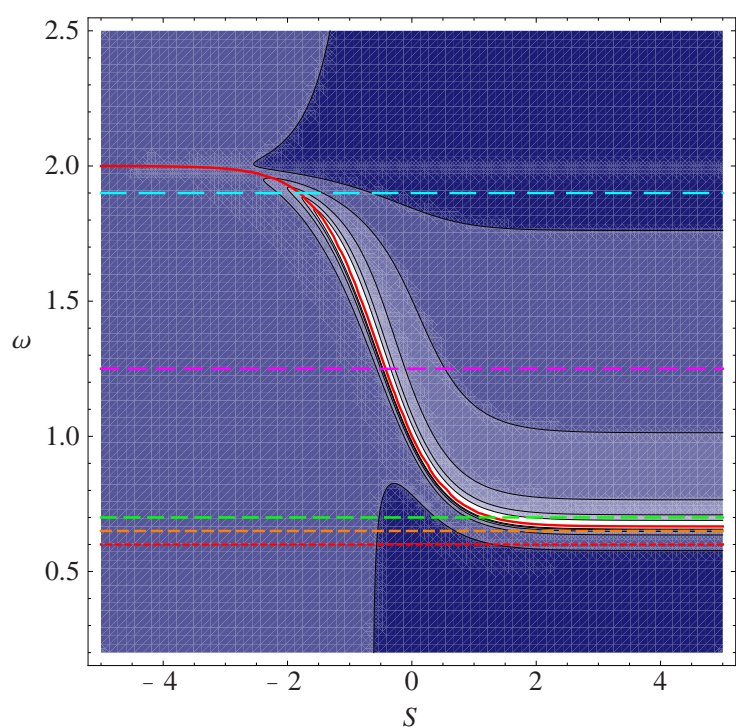

(a)

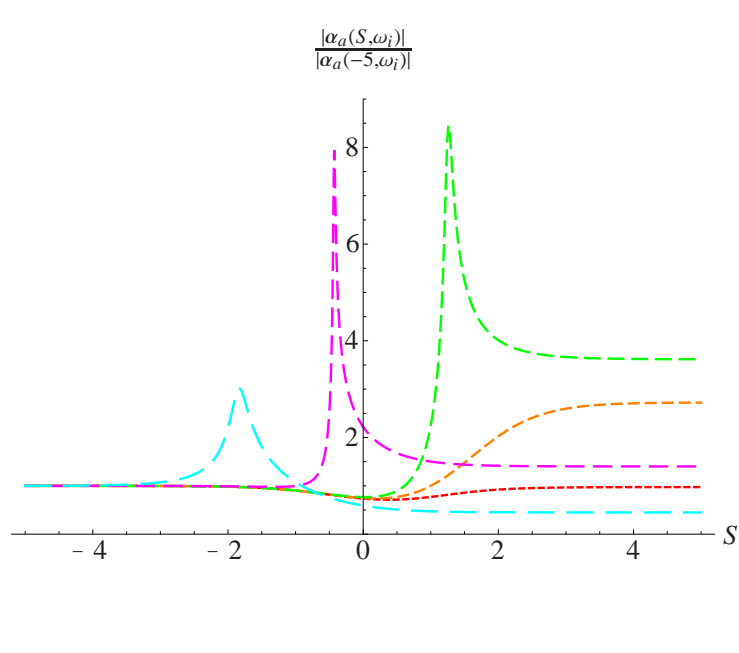

(b)

Figure 10: The contour plot in (a) gives an overall impression of how the amplitude of the modal vector is distributed over the frequency and slow coordinate. Plot (b) more precisely shows the level of the amplitude as function of the slow coordinate at the fixed frequencies indicated in the contour plot. In both plots the modal vector begins at a unit vector at $S=-5$

So far the results only differentiate themselves from classical cylindrical shell theory by evaluating the local value of the radius function. However, the real power of the WKB solution is its ability to also determine how the amplitude, e.g. expressed by $\left|\boldsymbol{\alpha}_{a}\right|$, is affected by the varying radius. The contours of the amplitude of the 1 st wave is plotted in figure 10(a) and at fixed frequencies in (b). It should be emphasised that the amplitude in both (a) and (b) is normalised such that at $S=-5$ it has unit magnitude at any frequency. Also, in the contour plot it should be remarked that the contour level is chopped such that the purely white narrow area in this plot corresponds to values of $\frac{\left|\alpha_{a}(S, \omega)\right|}{\left|\boldsymbol{\alpha}_{a}(-5, \omega)\right|}>5$. As the 1st wave is travelling along the shell, both plot (a) and (b) illustrate a rather dramatic evolution of the amplitude. Not only does the model predict the evolution of the amplitude to be very sensitive to the choice of fixed frequency, but also as soon as the wave comes near the location where the radius matches the ring-frequency a strong increase in amplitude appears. However, as the wave travels on and the radius changes even further, and thus moves away from the ring-frequency, the amplitude decrease again. This is because of the above mentioned localised breathing resonance. Having in mind that, as illustrated in figure 6(b), the coupled axial/breathing wave will inevitably contain some degree of breathing motion both before and after the mode conversion, then it will always have the potential to excite this localised breathing resonance. Hence the excitation mechanism of the resonance does not in principle rely on the mode conversion itself, but only depends on the breathing motion being present at the right location. This 
principle will be further discussed in subsection 3.8, where some of the properties of the waves related to $m=1$ are presented. Nevertheless, as the contour plots in figure 5 also show that the modal unit vector of the 1 st wave does in fact become dominated by its third component just before the ring-frequency, the displacement right at the amplitude peak will, in this case, consist above all of breathing motion.

Despite the apparent sharpness of the amplitude peaks it should finally be mentioned that the amplitude function is in fact smooth such that its derivative is continuous everywhere. Thus if the graphs were plotted against the fast coordinate instead, it would just be a matter of choosing a sufficiently small value of $\epsilon$ to spread out the peaks over a broader region.

\subsection{Energy Distribution - Shell (a)}

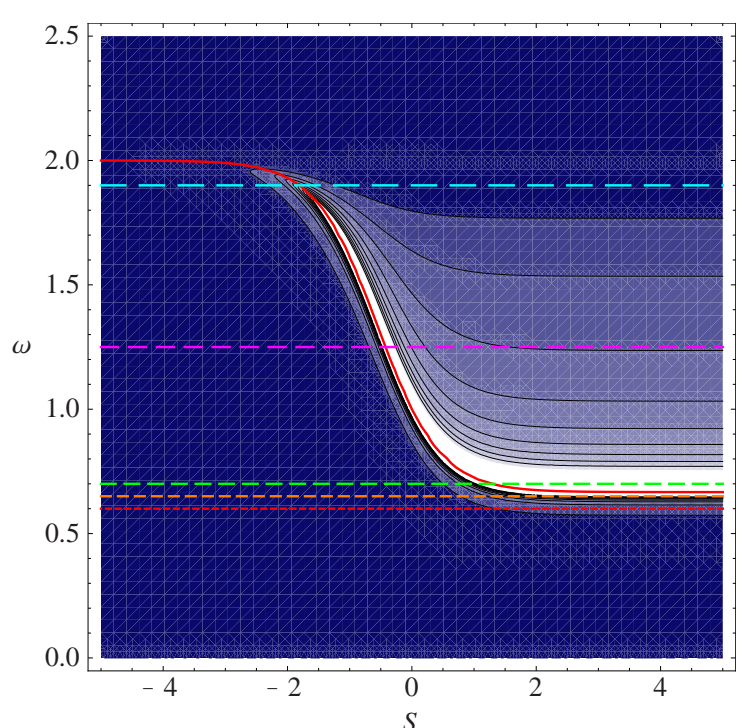

(a)

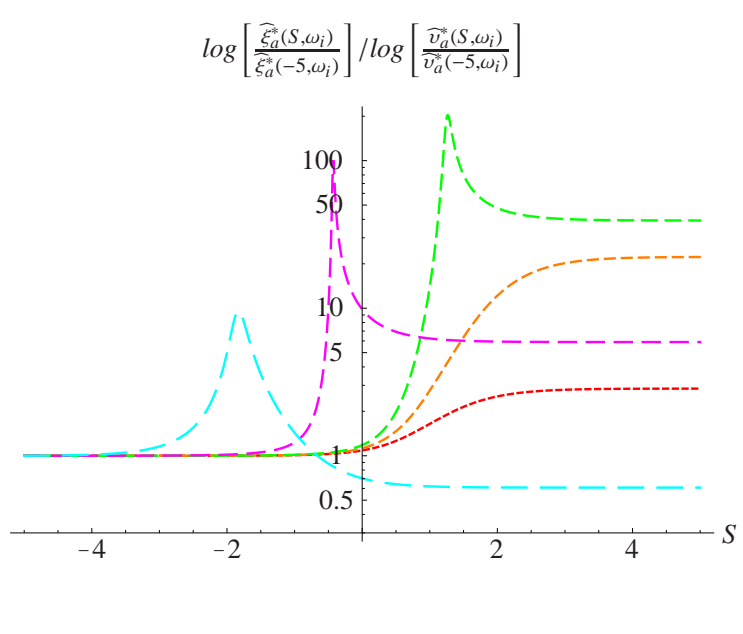

(b)

Figure 11: The contour plot in (a) gives an overall impression of how the kinetic/potential energy is distributed over the frequency and slow coordinate. Plot (b) more precisely shows the level of the kinetic/potential energy as function of the slow coordinate at the fixed frequency indicated in the contour plot. In both plots the energy has it unit magnitude at $S=-5$

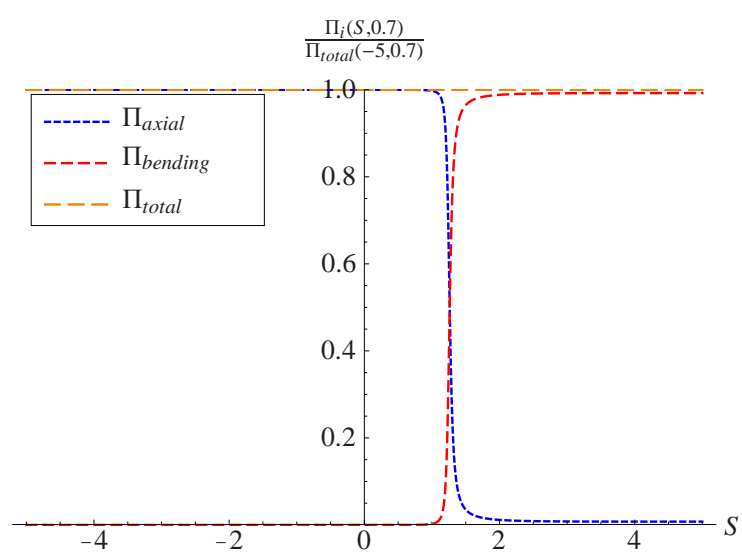

Figure 12: Total power flow as a results of axial and bending motion.

While the total power flow related to the axial/breathing mode has been proven to be conserved, the kinetic and potential energy may not be uniformly distributed throughout the shell, as explained in section 2.3. This is illustrated in figure 11, where the energy clearly is accumulated around the local resonance. In (a), the contour levels above 
20 are chopped and replaced by the purely white area. Plot (b) demonstrates how the energy level can be several decades higher at the local resonance (notice the logarithmic scale on the vertical axis), and, like the amplitude, the localisation of energy is very sensitive to the frequency. Returning to the power flow, it turns out that the total power, not surprisingly, is carried by different types of motion before and after the mode conversion. Thus in figure 12 the total power flow for $\omega=0.7$ is plotted together with its components of, respectively, axial and bending power. It confirms that in this particular case the power of the wave is carried by axial motion before the mode conversion and primarily by bending motion after the mode conversion.

\subsection{Dispersion Relation - Shell (b)}

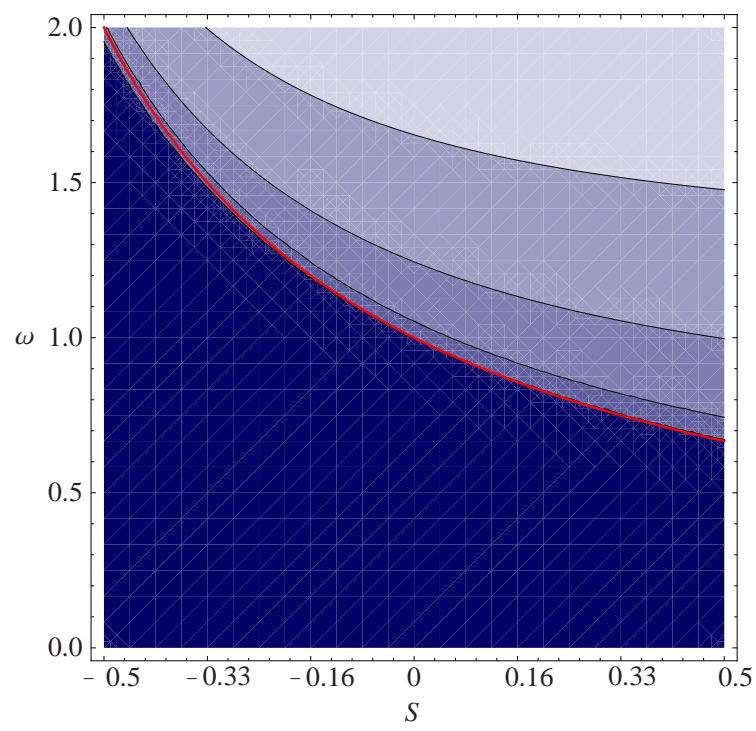

(a)

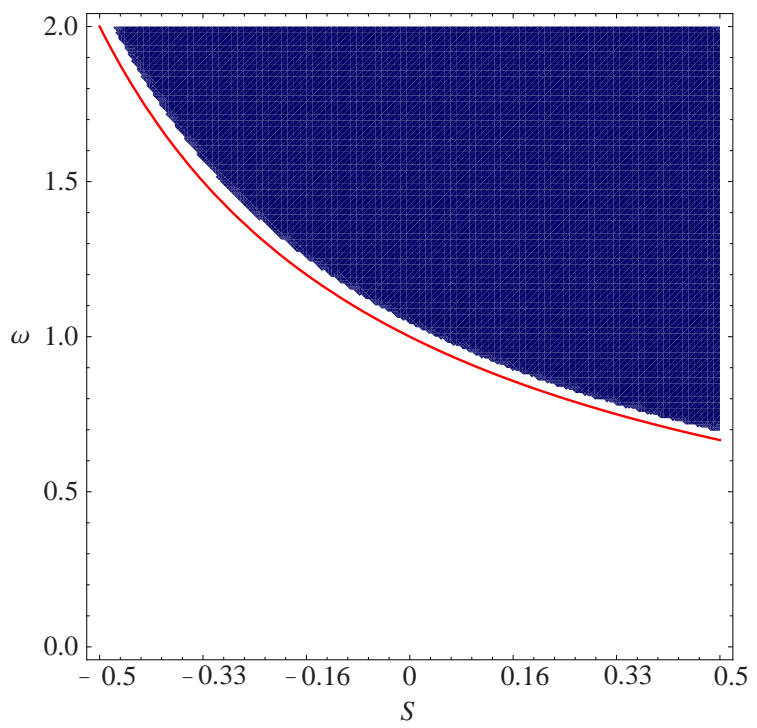

(b)

Figure 13: Contour plot of the wavenumber of (a) the 1st axial/breathing wave and (b) the 2nd axial/breathing wave.

For the conical shell the dispersion relation in principle behaves in much the same way as it does for shell (a), although because of the different radius function the contours in figure 13 are a different shaped to those in figure 3. Again the ring-frequency as a function of the slow coordinated is plotted with the red curve and indicates where the veering and mode conversion takes place. Because shell (a) consists of two uniform cylinders which are smoothly connected it is seen from the contour plots in figure 3 how the wavenumber and ring-frequency sufficiently far from $S=0$ become independent of the location. For shell (b), on the other hand, the radius changes linearly with location, and thus the ring-frequency, as well as the region of veering, vary even at large $S$.

\subsection{Modal Vector - Shell (b)}

The modal unit vector behaves in a similar way to shell (a). For simplicity the contour plot of the modal coefficients related to the 1 st wave are presented in figure 14 and plotted at a fixed frequency in figure 15, while the corresponding plots related to the 2 nd wave are omitted. Again the modal unit vector of the 1 st wave changes from being dominated by axial motion before the ring-frequency to being dominated by normal motion after the ring-frequency, whereas the modal unit vector of the 2 nd wave does the opposite right after the ring-frequency where it cuts on.

\subsection{Amplitude - Shell (b)}

Regarding the amplitude in figure 16, a peak exactly at the ring-frequency is present in shell (b). This confirms that the Gaussian curvature only has a minor, if any, effect on the amplitude and that the excitation of a localised breathing resonance is primarily causing the peak. Nevertheless it is probably in this plot the biggest difference from shell (a) is seen. Because the radius of the conical shell continues to change throughout its length the amplitude has a slightly negative slope both before and after the peak, whereas in shell (a) it finds a constant level sufficiently far from the peak. 


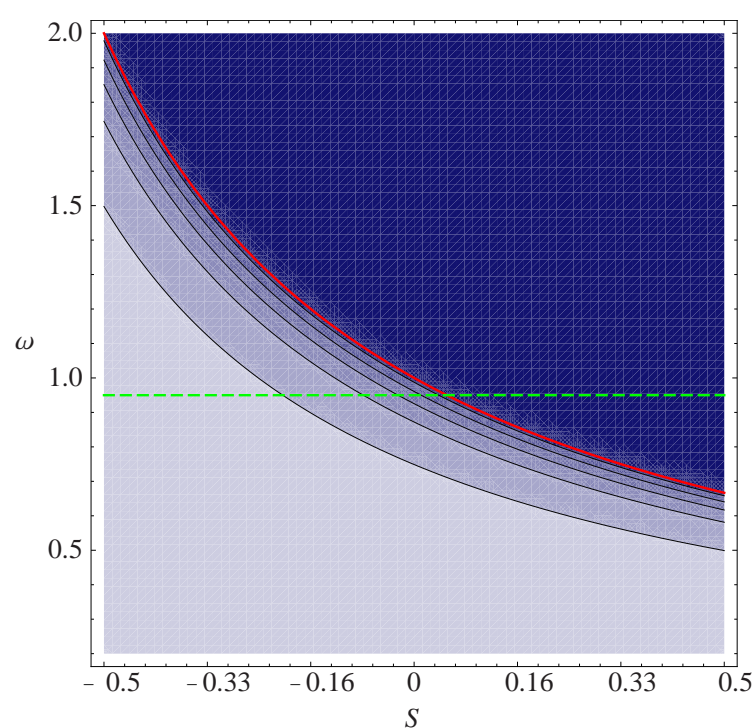

(a)

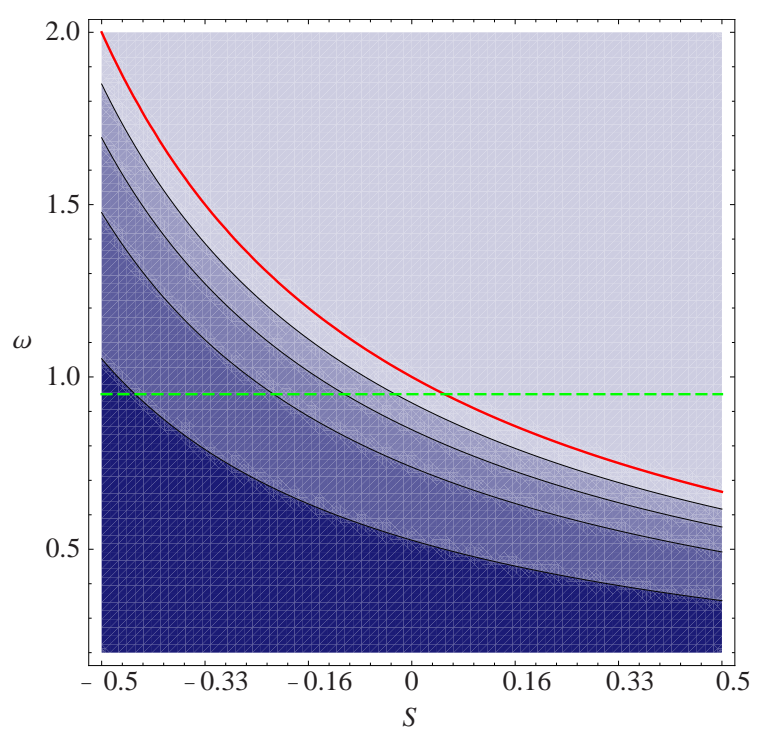

(b)

Figure 14: Contour plots of (a) the first component of the modal unit vector related to the 1st axial/breathing wave, i.e. $\frac{\left|\alpha_{a}(S, \omega)\right|}{\left|\alpha_{a}(S, \omega)\right|}$ and (b) the third component, i.e. $\frac{\left|\gamma_{a}(S, \omega)\right|}{\left|\boldsymbol{\alpha}_{a}(S, \omega)\right|}$.

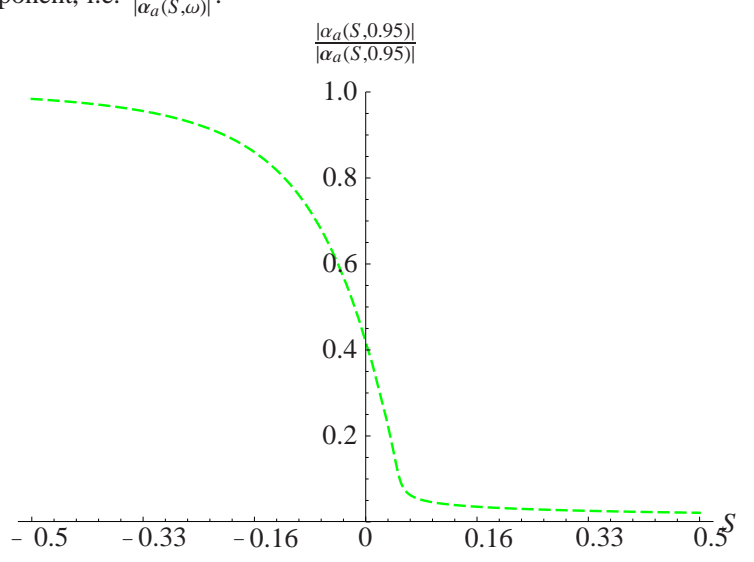

(a)

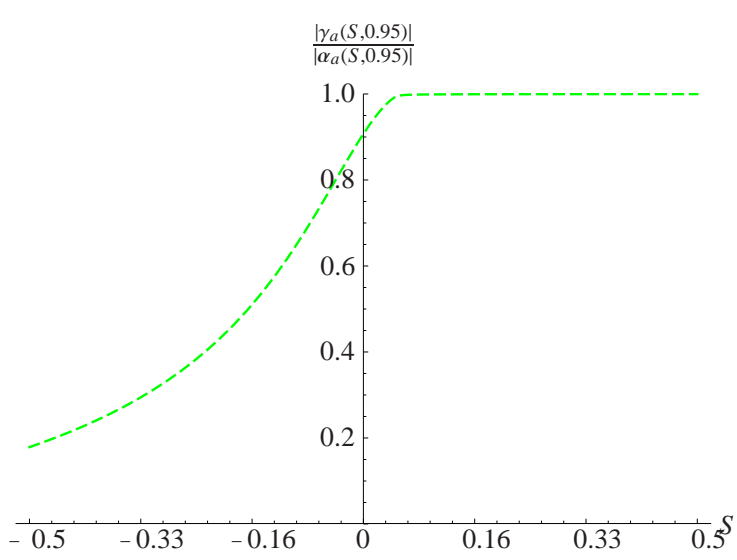

(b)

Figure 15: Along the fixed frequencies indicated by the dashed lines in figure 14, the components of the modal unit vector are plotted as function of the slow coordinate.

\subsection{When $m=1$ - The Bending Mode in Shell (a)}

The last example is related to the circumferential wavenumber $m=1$ and the corresponding waves travelling in shell (a). The following results are obtained from the numerical implementation briefly described in section 2.1.5.

\subsubsection{Dispersion Relation $-m=1$}

In figure 17 three plots of the dispersion relation are given. Figure 17(a) shows the wavenumbers of the three waves which are supported by the structure at $m=1$, as a function of the frequency. However, as for the axial/breathing wave, figure 17(b) and (c) demonstrate how the three waves are not all able to travel at any location along the shell. Obviously figure 17 (b) is reminiscent of figure 4(b), in the sense that the 1st wave is able to travel all the way along the shell, though due to the cut-on of the 2 nd wave and the following veering the wavenumber is changing rapidly in a localised region. However, at a frequency of $\omega=2.2$ figure 17(c) illustrates a slightly more complicated situation. At this frequency both the 1 st and the 2 nd waves are able to travel at any location along the shell, but due to the cut-on of a 3rd wave they are both forced to veer off. Like for the axial/breathing wave, the veering of the 1st wave in figure 


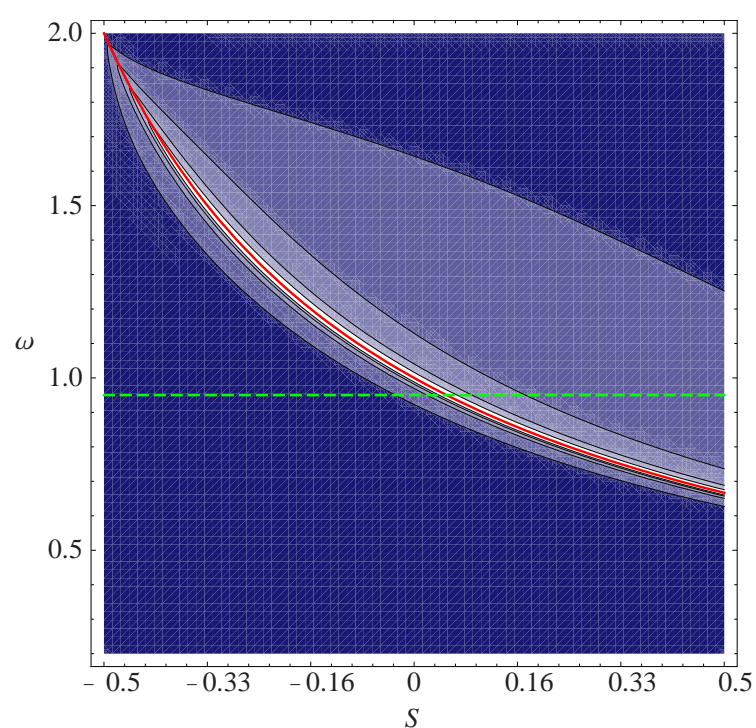

(a)

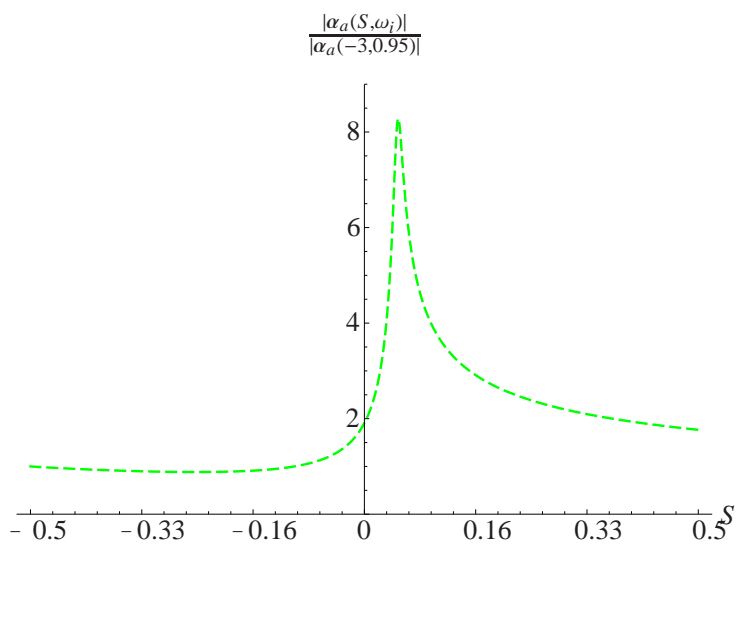

(b)

Figure 16: The contour plot in (a) gives an overall impression of how the amplitude of the modal vector is distributed over the frequency and slow coordinate. Plot (b) more precisely shows the level of the amplitude as function of the slow coordinate at the fixed frequencies indicated in the contour plot. In both plots the modal vector begins at a unit vector at $S=-5$

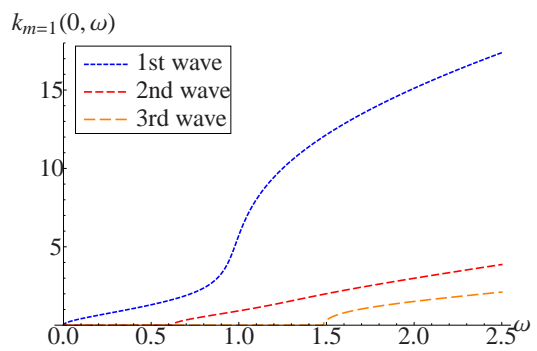

(a)

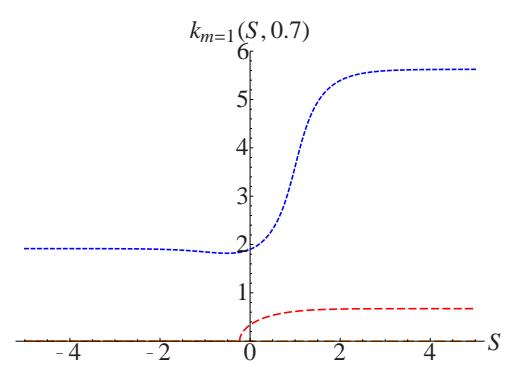

(b)

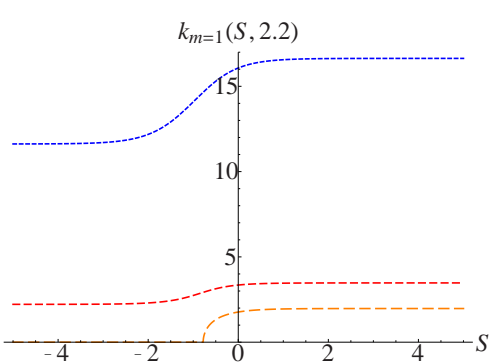

(c)

Figure 17: The wavenumber of the waves related to $m=1$ are plotted as function of (a) the frequency at $S=0$, (b) the slow coordinate at $\omega=0.7$, and (c) the slow coordinate at $\omega=2.2$

17(b) and the 1st and the 2nd waves in figure 17(c) is followed up by a mode conversion and possibly also a local resonance. To clarify this it is necessary to study how the modal vector and amplitude depend on location.

\subsubsection{Modal Vector and Amplitude $-m=1$}

As soon as $m \neq 0$ the three components of the modal vector are all coupled, c.f. equation (8), and must therefore be considered all together. The contour plot of the axial, circumferential, and normal component of the modal unit vector associated with the 1 st wave are presented in figure 18 and are plotted together as functions of the slow coordinate at, respectively, $\omega=0.7$ and $\omega=2.2$ in figure 19. The contour plots give an overview of how a mode conversion occurs due to the veering of the 1st wave. Qualitatively the s-shaped contour lines are reminiscent of those in figure 5 which, again, is due to the evolution of the radius function of shell (a). In figure 19(a) it can be seen that at the narrow end of shell (a) the 1st wave consists of an almost equal amount of circumferential and normal motion along with a smaller amount of axial motion. With reference to classical cylindrical shell theory, for $m=1$ this relation between the modal coefficients corresponds more or less to global bending. For the ideal situation the deformation of the cross-section of the shell is illustrated in the $(v, w)$-plan in figure 20(a). It can be shown that the relation between the modal coefficients in this ideal case is $\beta=\gamma=\frac{\alpha}{r(S) k(S)}$, and in practice for very low frequencies, that is $\omega<0.1$, this is obeyed. However, as this bending-like mode is travelling towards the wider end of shell (a), figure 19(a) show how it suddenly converts 


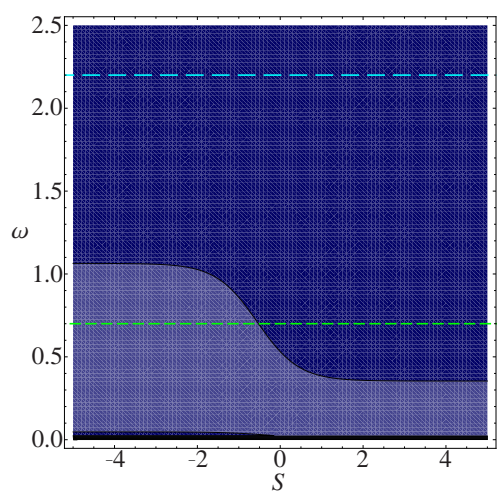

(a)

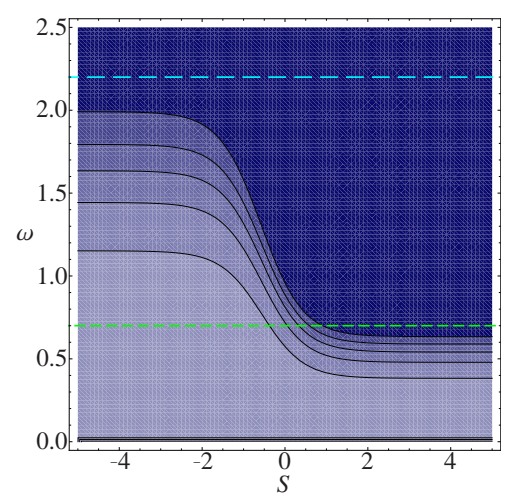

(b)

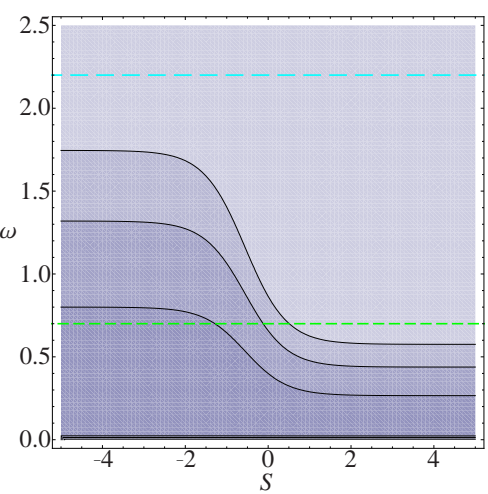

(c)

Figure 18: Contour plots of (a) the first component of the modal unit vector related to 1 st wave when $m=1$, i.e. $\frac{\left|\alpha_{1 s t}(S, \omega)\right|}{\left|\alpha_{1 s t}(S, \omega)\right|},($ b) the second component, $\frac{\left|\beta_{1 s t}(S, \omega)\right|}{\left|\alpha_{1 s t}(S, \omega)\right|}$, and (c) third component, $\frac{\left|\gamma_{1 s t}(S, \omega)\right|}{\left|\alpha_{1 s t}(S, \omega)\right|}$.

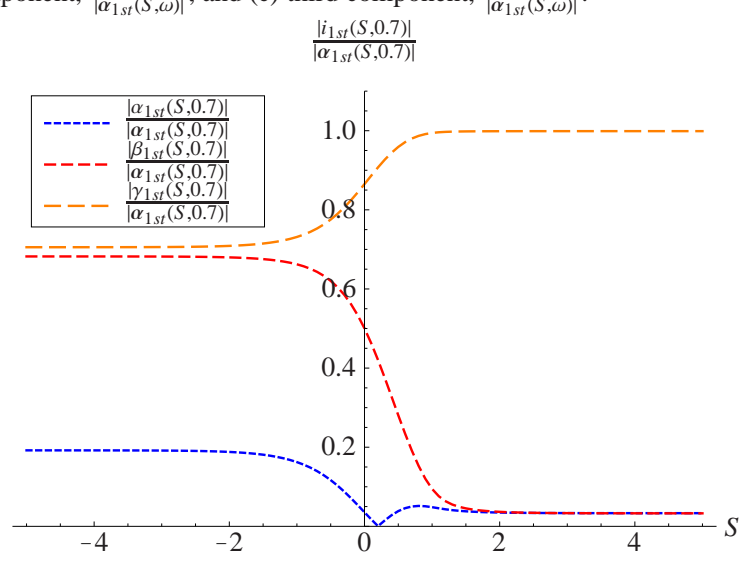

(a)

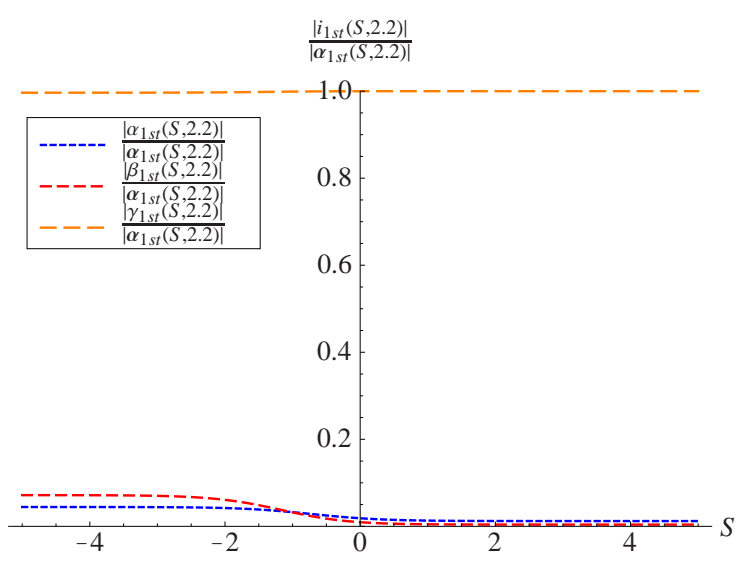

(b)

Figure 19: The three components of the modal unit vector plotted as function of the slow coordinate at (a) $\omega=0.7$ and (b) at $\omega=2.2$.

into a mode dominated purely by normal motion. The cross-section deformation of such a mode is sketched in figure 20(b). At $\omega=2.2$, figure 19(b) show how a mode similar to the one illustrated in figure 20(b) now dominates all the way along the shell. Nevertheless, due to the cut-on of the 3rd wave a slight conversion in the relation between the three modal coefficients still occurs.

Regarding the corresponding amplitude of the 1st wave, its contour plot is presented in figure 21(a) and plotted for the fixed frequencies indicated in the contour plot in figure 21(b). From these plots it is clear that for $m=1$ it also happens that a local resonance is exited, although the maximum amplification is not as high as for the axial/breathing wave. Again in this case, like for the axial/breathing wave, by comparing the amplitude evolution for $\omega=0.7$ and $\omega=2.2$ it is tempting to conclude that a more pronounced mode conversion is inevitably accompanied by an excitation of a localised resonance. However, it must be emphasised that this cannot be regarded as a general rule. To show this the modal coefficients related to the 2 nd wave are plotted in figure 22(a) at $\omega=2.2$. Obviously a strong modal conversion takes place as this wave is travelling along the shell. But in this case, as seen in figure 22(b), the corresponding amplitude show no sign of excitation of a localised resonance. Hence, in order to have an excitation of a local resonance it is first necessary to have a localised decrease in dynamic stiffness, and second the mode shape of the wave at that location needs to contain the components which are able to excite the resonance. Moreover, in relation to equation (8) this means that it is not enough only to consider the modal coefficients obtained by the $O(1)$-system, but the amplitude obtained by the $O(\epsilon)$-system must also be considered in order to predict the excitation of a local resonance. 


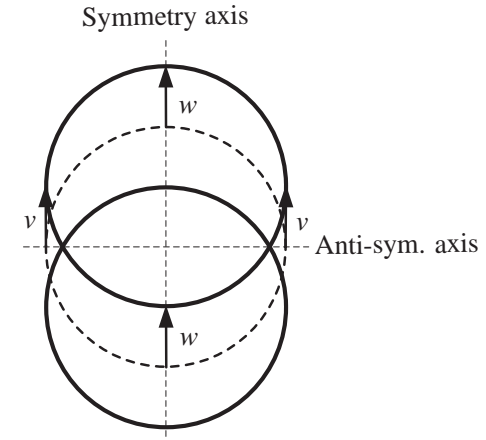

(a)

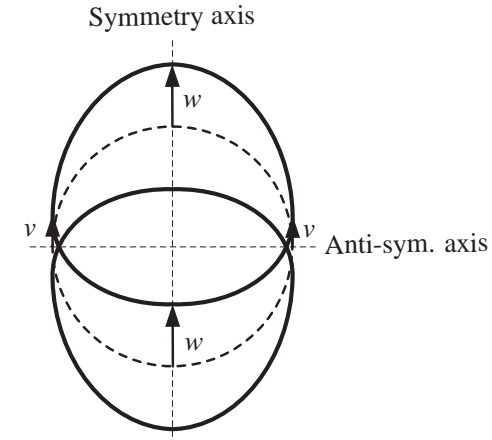

(b)

Figure 20: At low frequencies the cross-section of the shell moves as a rigid body (a), whereas it at higher frequencies converts to the deformation mode sketched in (b).

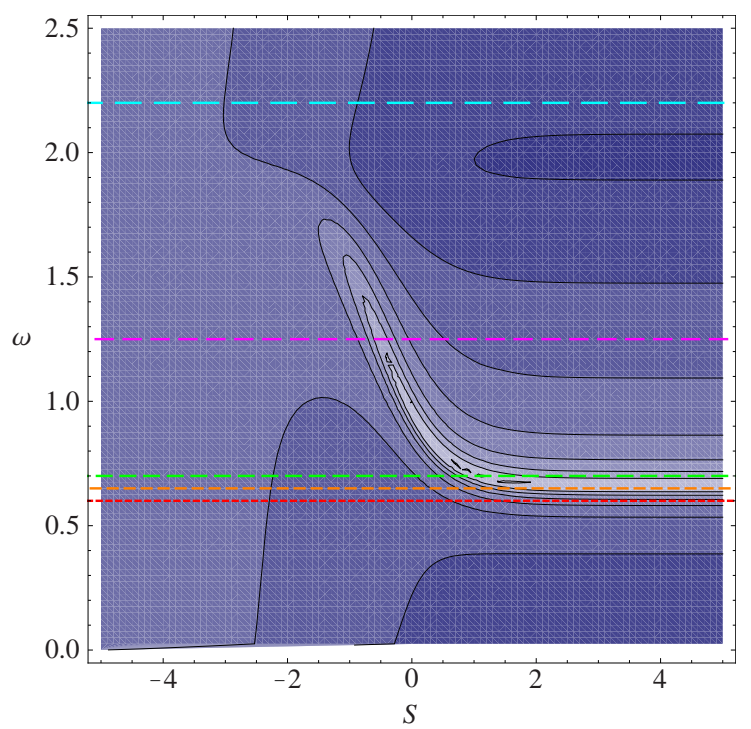

(a)

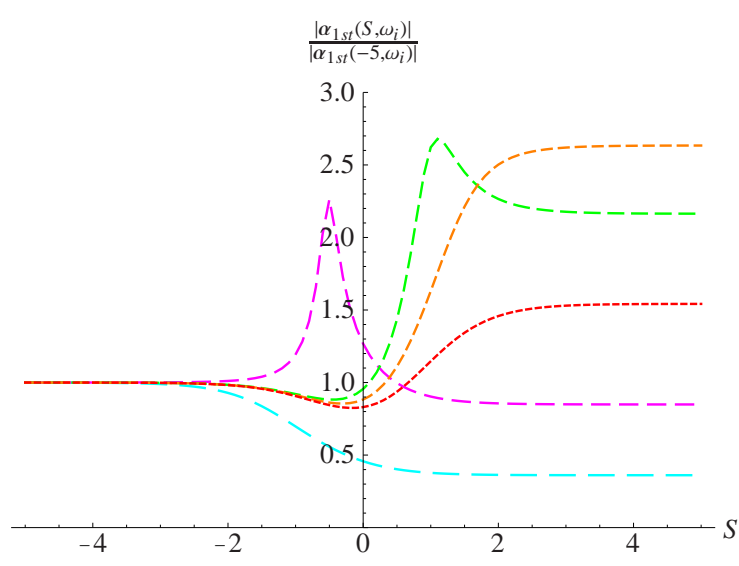

(b)

Figure 21: In (a) the contour the amplitude of 1st wave is plotted as function of the frequency and slow coordinate while it in (b) is plotted at the fixed frequencies indicated in (a).

\subsection{Final Remarks}

For the two selected shells, shell (a) and shell (b), it may be noticed that in both cases the radius function is monotonically increasing. On top of this, in the way the results are presented above, it may be naturally perceived as if the waves are travelling from $S=-\infty$ towards $S=\infty$. However, because both the wavenumber, modal vector, and amplitude are determined from the local properties, it makes no difference for these quantities in what direction the wave is travelling. The only quantity which is affected by the history of the waveguide is the phase, c.f. the integral formulation of the WKB solution in equation (7). Therefore, if the radius function is expressed via e.g. a Gauss distribution with its maxima at $S=0$, then the wave, except for its phase, will be identical at $S= \pm S_{0}$.

\section{Conclusions}

In this paper the waveguide properties of a shell of revolution have been examined. Firstly, the equations governing the elastodynamics of such a shell have been derived analytically. Under the assumption that the radius is a slowly 


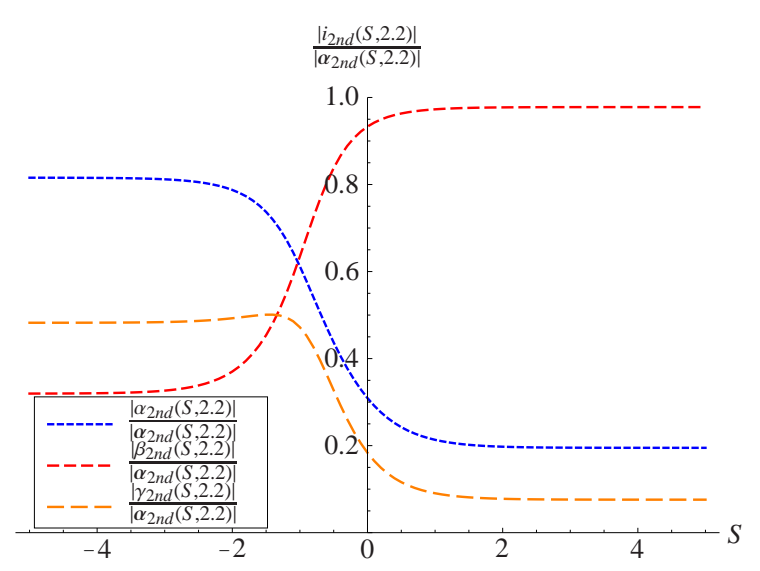

(a)

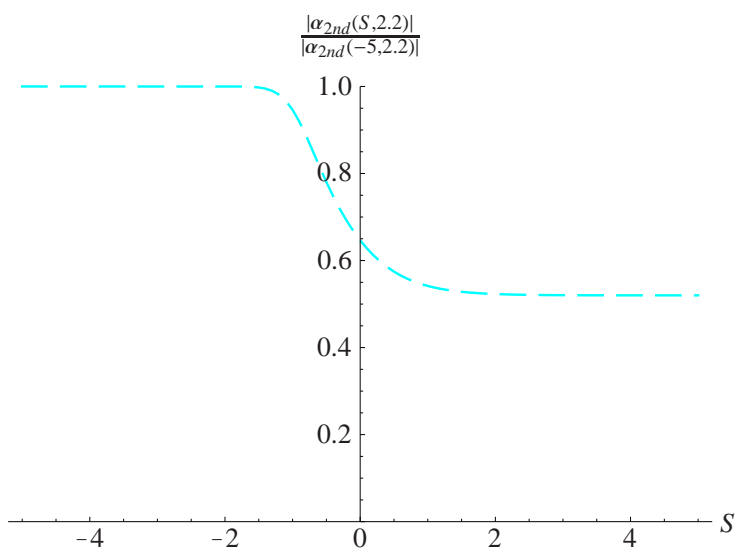

(b)

Figure 22: At a frequency of $\omega=2.2$, plot (a) illustrates the three components of the modal unit vector related to 2 nd wave as function of the slow coordinate and (b) the corresponding amplitude.

changing function of the axial coordinate, the governing equations have been asymptotically approximated, and then solved by means of the WKB-method. With this solution method the dispersion relation and the modal vector are determined from the $O(1)$-equations, that is the leading order equations, as if the shell was a uniform cylinder with the local properties. In contrast, the effects of the changing radius are accumulated in the amplitude which, by considering the $O(\epsilon)$-equations, are formulated in an integral form, see equation (14). For the circumferential wavenumber $m=0$ the integration has been done analytically, and hence the dependency of the amplitude on the radius function for the fundamental modes, i.e. the torsional wave and the axial/breathing wave, have been obtained in closed form, see equations (19), (23), and (24). However, for arbitrary $m$, even with state-of-the-art symbolic computation software, it has not been found possible to carry out the integration analytically. Instead, by specifying a radius function, the evolution of the amplitude, for each specific wave, has been successfully obtained by numerical integration.

The validity of the analytical expressions has been tested by showing that the power flow associated with the analytical solution is conserved. Moreover it has been demonstrated that, even though the time-averaged kinetic and potential energy have a non-uniform distribution throughout the shell, they are still of the same magnitude at any location. This is a prerequisite for a conservative elastodynamical system. As a by-product of these considerations it becomes easy to determine to what extent the wave energy is carried in the shell wall by axial motion, shearing motion or bending motion, and how the contribution of each type of motion may gradually change with the evolution of the radius function.

Despite the complexity of the shell structure, the properties of the torsional wave and the decoupled axial/breathing wave have been related to classical rod and beam theory and show an excellent correlation. On one hand this also confirms the validity of the derived equations, but, just as importantly, on the other hand it points out a short-cut to predict, under suitable conditions, the behaviour of these fundamental waves supported by the shell of revolution.

To further demonstrate the capabilities of the derived model and solution, and to illustrate some general waveguide properties of shells of revolution, two characteristic shapes of shells have been specified. For these shells it is explained how high-order modes cut on, one after one, as the radius increases, and how veering of coupled waves is taking place, not only as a function of frequency, but also as function of location along the shell. Along with the veering it has also been demonstrated how the modal vector is changed such that a wave travelling along the shell may change its mode shape completely over a rather short distance. Specifically, for the axial/breathing wave it is shown how the veering and mode conversion is triggered by the match between the frequency of the travelling wave, the local radius and the ring-frequency given by $\omega_{r}=r(S)^{-1}$. On top of this, in the vicinity of where the conditions for the ring-frequency set in a localised resonance appears, making the shell sensitive to breathing motion. Consequently a very large increase in breathing motion appears in this region. Finally, when examining how a set of higher-order waves depend on the changing radius, it becomes clear that, on one hand, the mode conversion of such waves can also be accompanied by an excitation of a localised resonance. On the other hand, examples have also been found where a pronounced mode conversion is taking place, but without any noticeable increase in amplitude. Hence, the location of a localised peak 
amplitude cannot be indirectly predicted from the mode conversion obtained purely by the $O(1)$-equations, but needs the information from the $O(\epsilon)$-equations in order to explicitly determine the amplitude.

For future work a reasonable next step is to compare these rigorously derived analytical results with numerical models such as a finite element model. Likewise, experimental validation would also be highly appreciated. Possible extensions of the current model could be to include fluid interaction, either inside or outside or both. It is expected that the peaks in amplitude at localised resonances will still be present, but they will probably be reduced in magnitude because the fluid will provide a way to dissipate energy from these areas. Another extension to the model could also be to consider a bounded waveguide. This will involve the generalised forces in equation (32) which e.g. must be zero for a free boundary or may be used to calculate the reactions at a restricted or even clamped boundary.

\section{Acknowledgements}

This research has been founded by The Danish Council for Technology and Innovation, under grand no. 10-083896, which is gratefully acknowledged.

\section{Appendix A Equations of Motion Expressed in Displacements}

The equations of motion expressed in displacements of the shell of revolution are obtained by removing the circumferential dependency from the Lamé parameters and radii of curvatures from the general shell equations. Thus the displacement-deformation relations for a shell of revolution are

$$
\begin{aligned}
& \varepsilon_{s}=\frac{1}{A_{s}^{*}} \frac{\partial u^{*}}{\partial s}+\frac{1}{R_{s}^{*}} w^{*} \\
& \varepsilon_{\theta}=\frac{1}{A_{s}^{*} A_{\theta}^{*}} \frac{\partial A_{\theta}^{*}}{\partial s} u^{*}+\frac{1}{A_{\theta}^{*}} \frac{\partial v^{*}}{\partial \theta}+\frac{1}{R_{\theta}^{*}} w^{*} \\
& \gamma=\frac{1}{A_{\theta}^{*}} \frac{\partial u^{*}}{\partial \theta}+\frac{A_{\theta}^{*}}{A_{s}^{*}} \frac{\partial}{\partial s}\left(\frac{v^{*}}{A_{\theta}^{*}}\right) \\
& \kappa_{s}^{*}=\frac{1}{A_{s}^{*}} \frac{\partial}{\partial s}\left(\frac{1}{R_{s}^{*}} u^{*}-\frac{1}{A_{s}^{*}} \frac{\partial w^{*}}{\partial s}\right) \\
& \kappa_{\theta}^{*}=\frac{1}{A_{s}^{*} A_{\theta}^{*} R_{s}^{*}} \frac{\partial A_{\theta}^{*}}{\partial s} u^{*}+\frac{1}{A_{\theta}^{*} R_{\theta}^{*}} \frac{\partial v^{*}}{\partial \theta}-\frac{1}{A_{\theta}^{* 2}} \frac{\partial^{2} w^{*}}{\partial \theta^{2}}-\frac{1}{A_{s}^{* 2} A_{\theta}^{*}} \frac{\partial A_{\theta}^{*}}{\partial s} \frac{\partial w^{*}}{\partial s} \\
& \tau^{*}=\frac{1}{A_{\theta}^{*} R_{s}^{*}} \frac{\partial u^{*}}{\partial \theta}+\frac{1}{R_{\theta}^{*}}\left(\frac{1}{A_{s}^{*}} \frac{\partial v^{*}}{\partial s}-\frac{1}{A_{s}^{*} A_{\theta}^{*}} \frac{\partial A_{\theta}^{*}}{\partial s} v^{*}\right)-\frac{1}{A_{s}^{*} A_{\theta}^{*}}\left(\frac{\partial^{2} w^{*}}{\partial s \partial \theta}-\frac{1}{A_{\theta}^{*}} \frac{\partial A_{\theta}^{*}}{\partial s} \frac{\partial w^{*}}{\partial \theta}\right),
\end{aligned}
$$

where $\varepsilon_{i}$ is the membrane normal strain in direction $i, \gamma$ is the membrane shear strain, $\kappa_{i}^{*}$ is the change of curvature in direction $i$, and $\tau^{*}$ is the twist. Likewise, the constitutive relations are 


$$
\begin{aligned}
& T_{s}^{*}=\frac{E^{*} h^{*}}{1-v^{2}}\left(\varepsilon_{s}+v \varepsilon_{\theta}\right) \\
& T_{\theta}^{*}=\frac{E^{*} h^{*}}{1-v^{2}}\left(\varepsilon_{\theta}+v \varepsilon_{s}\right) \\
& M_{s}^{*}=\frac{E^{*} h^{* 3}}{12\left(1-v^{2}\right)}\left(\kappa_{s}^{*}+v \kappa_{\theta}^{*}\right) \\
& M_{\theta}^{*}=\frac{E^{*} h^{* 3}}{12\left(1-v^{2}\right)}\left(\kappa_{\theta}^{*}+v \kappa_{s}^{*}\right) \\
& T_{s \theta}^{*}=\frac{E^{*} h^{*}}{2(1+v)}\left(\gamma+\frac{h^{* 2}}{6 R_{\theta}^{*}} \tau^{*}\right) \\
& T_{\theta s}^{*}=\frac{E^{*} h^{*}}{2(1+v)}\left(\gamma+\frac{h^{* 2}}{6 R_{s}^{*}} \tau^{*}\right) \\
& M_{s \theta}^{*}=M_{\theta s}^{*}=\frac{E^{*} h^{* 3}}{12(1+v)} \tau^{*},
\end{aligned}
$$

where $T_{i}^{*}$ is the membrane normal force resultant in direction $i, M_{i}^{*}$ is the bending moment resultant due to normal stresses in direction $i, T_{i j}^{*}$ is the membrane shear force resultant acting in direction $j$ on a surface normal to $i, M_{i j}^{*}$ is the torsion moment, $E^{*}$ is the modulus of elasticity, $v$ is Poisson's ratio, and $h^{*}$ is the thickness of the shell. The equations of motion, obtained by establishing the force balance, are

$$
\begin{aligned}
0 & =\frac{1}{A_{s}^{*} A_{\theta}^{*}}\left(\frac{\partial}{\partial s}\left(A_{\theta}^{*} T_{s}^{*}\right)+A_{s}^{*} \frac{\partial T_{\theta s}^{*}}{\partial \theta}-\frac{\partial A_{\theta}^{*}}{\partial s} T_{\theta}^{*}\right)+\frac{N_{s}^{*}}{R_{s}^{*}}-\rho^{*} h^{*} \frac{\partial^{2} u^{*}}{\partial t^{* 2}} \\
0 & =\frac{1}{A_{s}^{*} A_{\theta}^{*}}\left(\frac{\partial}{\partial s}\left(A_{\theta}^{*} T_{s \theta}^{*}\right)+A_{s}^{*} \frac{\partial T_{\theta}^{*}}{\partial \theta}+\frac{\partial A_{\theta}^{*}}{\partial s} T_{\theta s}^{*}\right)+\frac{N_{\theta}^{*}}{R_{\theta}^{*}}-\rho^{*} h^{*} \frac{\partial^{2} v^{*}}{\partial t^{* 2}} \\
0 & =-\frac{1}{A_{s}^{*} A_{\theta}^{*}}\left(\frac{\partial}{\partial s}\left(A_{\theta}^{*} N_{s}^{*}\right)+A_{s}^{*} \frac{\partial N_{\theta}^{*}}{\partial \theta}\right)+\frac{T_{s}^{*}}{R_{s}^{*}}+\frac{T_{\theta}^{*}}{R_{\theta}^{*}}+\rho^{*} h^{*} \frac{\partial^{2} w^{*}}{\partial t^{* 2}},
\end{aligned}
$$

where $N_{i}^{*}$ is the out-of-plane shear force resultant acting on a surface normal to $i, \rho^{*}$ is the density, and $t^{*}$ is the time. In this force balance the dynamics of the shell has been incorporated as an inertia force acting on the middle surface of the shell. Finally the force resultants $N_{i}^{*}$ are obtained by the moment balance

$$
\begin{aligned}
& N_{s}^{*}=\frac{1}{A_{s}^{*} A_{\theta}^{*}}\left(\frac{\partial}{\partial s}\left(A_{\theta}^{*} M_{s}^{*}\right)+A_{s}^{*} \frac{\partial M_{\theta s}^{*}}{\partial \theta}-\frac{\partial A_{\theta}^{*}}{\partial s} M_{\theta}^{*}\right) \\
& N_{\theta}^{*}=\frac{1}{A_{s}^{*} A_{\theta}^{*}}\left(\frac{\partial}{\partial s}\left(A_{\theta}^{*} M_{s \theta}^{*}\right)+A_{s}^{*} \frac{\partial M_{\theta}^{*}}{\partial \theta}+\frac{\partial A_{\theta}^{*}}{\partial s} M_{\theta s}^{*}\right) .
\end{aligned}
$$


By combining equation (39) to (42) the equations of motion are expressed purely in in terms of the shell displacements.

\section{Appendix B The Breathing Wave Modelled as an Euler-Bernoulli Beam on a Winkler Foundation}

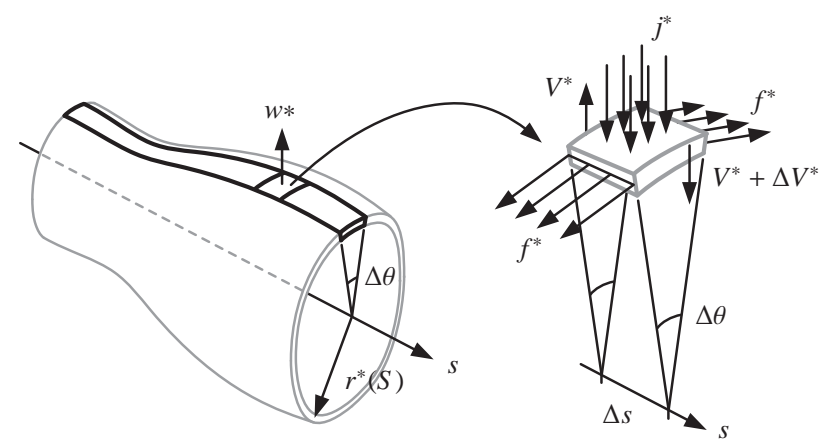

Figure 23: The breathing mode in the shell of revolution may be replicated by cutting out a narrow strip along a fixed angle $\Delta \theta$ and then model the bending behaviour while the strip is supported by a Winkler foundation.

When the shell of revolution is undergoing breathing motion, it is possible to replicate its behaviour by cutting out a narrow strip along a fixed small angle, see $\Delta \theta$ in figure 23, and then modelling the bending properties of this strip. By further cutting out a segment of dimensional length $\Delta s^{*}$, the edges facing the axial direction will, due to the bending, be loaded with moments, $M^{*}$, (though not illustrated) and out-of-plane shearing forces, $V^{*}$, which are related by $\frac{\partial M^{*}}{\partial s^{*}}=V^{*}$. Moreover, when the shell displaces uniformly along the circumference, in the normal direction, the shell will respond with membrane stresses, $f^{*}$, in the circumference which are acting on the strip as an elastic foundation, i.e. a Winkler foundation. The level of these stresses are controlled by the straining of the circumference. Hence it may be found that $f^{*}=E^{*} \frac{w^{*}}{r^{*}(S)}$. Finally due to the mass of the segment it will be affected by translational inertia, $j^{*}$, distributed over its volume. Thus the forcing balance projected on the $w$-axis is

$$
\begin{aligned}
0 & =V^{*}-\left(V^{*}+\Delta V^{*}\right)-2 E^{*} \frac{w^{*}}{r^{*}(S)} \sin \left(\frac{\Delta \theta}{2}\right) \Delta s^{*} h^{*}-\rho^{*} \Delta s^{*} r^{*}(S) \Delta \theta h^{*} \frac{\partial^{2} w^{*}}{\partial t^{* 2}} \\
& \simeq-\Delta V^{*}-E^{*} \frac{w^{*}}{r^{*}(S)} h^{*} \Delta \theta \Delta s^{*}-\rho^{*} r^{*}(S) \Delta \theta h^{*} \frac{\partial^{2} w^{*}}{\partial t^{* 2}} \Delta s^{*} .
\end{aligned}
$$

Dividing by $\Delta s^{*}$ and taking the limit of $\Delta s^{*} \rightarrow 0$ yields

$$
\begin{aligned}
0 & =-\frac{\partial V^{*}}{\partial s^{*}}-E^{*} \frac{w^{*}}{r^{*}(S)} h^{*} \Delta \theta-\rho^{*} r^{*}(S) \Delta \theta h^{*} \frac{\partial^{2} w^{*}}{\partial t^{* 2}}= \\
& =-\frac{\partial^{2} M^{*}}{\partial s^{* 2}}-E^{*} \frac{w^{*}}{r^{*}(S)} h^{*} \Delta \theta-\rho^{*} r^{*}(S) \Delta \theta h^{*} \frac{\partial^{2} w^{*}}{\partial t^{* 2}} .
\end{aligned}
$$

Now, as well as the shell in this paper being subjected to the Kirchhoff-Love assumptions, this strip may be regarded as an Euler-Bernoulli beam. Thus the relation between the bending moment and the displacement is given by the Euler-Bernoulli beam equation $M^{*}=E^{*} I^{*} \frac{\partial^{2} w^{*}}{\partial s^{* 2}}$ where $I^{*}$ is the area moment of inertia of the strip. And because the strip is narrow, the area moment of inertia can simply be expressed as $I^{*}(S)=\frac{1}{12} r^{*}(S) \Delta \theta h^{* 3}$. Then, using the time dependency $w^{*}\left(s, t^{*}\right)=r_{0}^{*} \tilde{w}(s) \operatorname{Exp}\left(\frac{i \omega c^{*} t^{*}}{r_{0}^{*}}\right)$, and scaling lengths with $r_{0}^{*}$ the PDE from above simplifies to

$$
0=\frac{\delta^{2}}{12} \frac{\partial^{2}}{\partial s^{2}}\left(r(S) \frac{\partial^{2} \tilde{w}}{\partial s^{2}}\right)+\left(\frac{1}{r(S)}-r(S) \omega^{2}\right) \tilde{w} .
$$




\section{Reference List}

[1] A. L. Gol'denweizer, V. B. Lidskij, P. E. Tovstik, Free Vibration of Thin Elastic Shells, Moskva: Nauka, 1979, in Russian.

[2] S. V. Sorokin, Influence of Small Imperfections of Shape on Natural Frequencies of Shells of Revolution, PhD Thesis, Leningrad Shipbuilding Institute, Leningrad, 1981, in Russian.

[3] V. D. Kubenko, P. S. Kovalchuk, T. S. Krasnopolskaja, Non-linear Interaction of the Bending Vibrations Modes of Cylindrical Shells, Naukova Dumka, Kiev, 1984, in Russian.

[4] Y. P. Guo, Normal mode propagation on conical shells, The Journal of the Acoustical Society of America, 1994, vol. 96, no. 1.

[5] D. Gridin, R. V. Craster, Quasimodes of a weakly curved waveguide, The Royal Society, 2003.

[6] D. Gridin, R. V. Craster, J. Fong, M. J. S. Lowe, M. Beard, The high-frequency asymptotic analysis of guided waves in a circular elastic annulus, Wave Motion, 2003.

[7] J. D. Kaplunov, L. Y. Kossovich, E. V. Nolde, Dynamics of thin walled elastic bodies, ACADEMIC PRESS, 1998.

[8] V. V. Novozhilov, The Theory Of Thin Shells, P. Noordhoff LTD., 1959.

[9] W. Flügge, Statik und Dynamik Der Schalen, Springer, 1934.

[10] J. Headning, An introduction to phase-integral methods, Methuen \& Company Ltd, 1962.

[11] S. S. Rao, Mechanical Vibrations, Pearson, 2004, 4th edition.

[12] L. Cremer, M.Heckl, B. A. T. Petersson, Structure-Borne Sound, Springer, 2005, 3rd edition.

[13] S. Sorokin, J. Nielsen, N. Olhoff, Greens matrix and the boundaryintegral equation method for the analysis of vibration and energy flow in cylindrical shells with and without internal fluid loading, Journal of Sound and Vibration, 2004, pp. 815-847.

[14] L. Rayleigh, The Theory of Sound, Republished in 1945 by Dover Publications, 1894.

[15] B. R. Mace, E. Manconi, Wave motion and dispersion phenomena: Veering, locking and strond coupling effects, Acoustical Society of America, 2012, pp.: 1015-1028 